\title{
Identification of a six-gene metabolic signature predicting overall survival for patients with lung adenocarcinoma
}

\author{
Yubo Cao ${ }^{\text {Corresp., } 1}$, Xiaomei $\mathrm{Lu}^{2}$, Yue $\mathrm{Li}{ }^{1}$, Jia Fu ${ }^{1}$, Hongyuan $\mathrm{Li}^{1}{ }^{1}$, Xiulin $\mathrm{Li}^{1}{ }^{1}$, Ziyou Chang ${ }^{1}$, Sa Liu ${ }^{\text {Corresp. } 1}$ \\ ${ }^{1}$ Department of Medical Oncology, The Fourth Affiliated Hospital of China Medical University, Shenyang, China \\ 2 Department of Pathophysiology, China Medical University, Shenyang, China \\ Corresponding Authors: Yubo Cao, Sa Liu \\ Email address: ybcao@cmu.edu.cn, liusa118@163.com
}

Background: Lung cancer is the leading cause of cancer-related deaths worldwide. Lung adenocarcinoma (LUAD) is one of the main subtypes of lung cancer. Hundreds of metabolic genes are altered consistently in LUAD; however, their prognostic role remains to be explored. This study aimed to establish a molecular signature that can predict the prognosis in patients with LUAD based on metabolic gene expression. Methods: The transcriptome expression profiles and corresponding clinical information of LUAD were obtained from the Cancer Genome Atlas and Gene Expression Omnibus databases. The differentially expressed genes (DEGs) between LUAD and paired non-tumor samples were identified by the Wilcoxon rank sum test. Univariate Cox regression analysis and the lasso Cox regression model were used to construct the best-prognosis molecular signature. $A$ nomogram was established comprising the prognostic model for predicting overall survival. To validate the prognostic ability of the molecular signature and the nomogram, the Kaplan-Meier survival analysis, Cox proportional hazards model, and receiver operating characteristic analysis were used. Results: The six-gene molecular signature (PFKP, PKM, TPI1, LDHA, PTGES, and TYMS) from the DEGs was constructed to predict the prognosis. The molecular signature demonstrated a robust independent prognostic ability in the training and validation sets. The nomogram including the prognostic model had a greater predictive accuracy than previous systems. Furthermore, a gene set enrichment analysis revealed several significantly enriched metabolic pathways, which suggests a correlation of the molecular signature with metabolic systems and may help explain the underlying mechanisms. Conclusions: Our study identified a novel six-gene metabolic signature for LUAD prognosis prediction. The molecular signature could reflect the dysregulated metabolic microenvironment, provide potential biomarkers for predicting prognosis, and indicate potential novel metabolic molecular-targeted therapies. 


\section{Identification of a six-gene metabolic signature}

3 predicting overall survival for patients with lung

4 adenocarcinoma

5

6

7

8

9

10

11

12

13

14

15

16

17

18

19

20

21

22

23

24

25

26

27

28

29

30

31

32

33

34

35

36
Yubo $\mathrm{Cao}^{1}$, Xiaomei $\mathrm{Lu}^{2}$, Yue $\mathrm{Li}^{1}$, Jia Fu${ }^{1}$, Hongyuan $\mathrm{Li}^{1}$, Xiulin $\mathrm{Li}^{1}$, Ziyou Chang ${ }^{1}$, Sa Liu ${ }^{1}$

${ }^{1}$ Department of Medical Oncology, The Fourth Affiliated Hospital of China Medical University, Shenyang, Liaoning, China

${ }^{2}$ Department of Pathophysiology, China Medical University, Shenyang, Liaoning, China

Corresponding Authors:

Yubo Cao

No.4 Chongshan East Road, Huanggu District, Shenyang, Liaoning, Zip code: 110032, China

Email address: ybcao@cmu.edu.cn

Sa Liu

No.4 Chongshan East Road, Huanggu District, Shenyang, Liaoning, Zip code: 110032, China

Email address: liusa118@163.com 


\section{Abstract}

38 Background: Lung cancer is the leading cause of cancer-related deaths worldwide. Lung

39

40

41

42

43

44

45

46

47

48

49

50

51

52

53

54

55

56

57

58

59

60

61

62

63

64

65

66

67

68

69

70

71

72

73

74

75

76

adenocarcinoma (LUAD) is one of the main subtypes of lung cancer. Hundreds of metabolic genes are altered consistently in LUAD; however, their prognostic role remains to be explored. This study aimed to establish a molecular signature that can predict the prognosis in patients with LUAD based on metabolic gene expression.

Methods: The transcriptome expression profiles and corresponding clinical information of LUAD were obtained from the Cancer Genome Atlas and Gene Expression Omnibus databases. The differentially expressed genes (DEGs) between LUAD and paired non-tumor samples were identified by the Wilcoxon rank sum test. Univariate Cox regression analysis and the lasso Cox regression model were used to construct the best-prognosis molecular signature. A nomogram was established comprising the prognostic model for predicting overall survival. To validate the prognostic ability of the molecular signature and the nomogram, the Kaplan-Meier survival analysis, Cox proportional hazards model, and receiver operating characteristic analysis were used.

Results: The six-gene molecular signature (PFKP, PKM, TPI1, LDHA, PTGES, and TYMS) from the DEGs was constructed to predict the prognosis. The molecular signature demonstrated a robust independent prognostic ability in the training and validation sets. The nomogram including the prognostic model had a greater predictive accuracy than previous systems. Furthermore, a gene set enrichment analysis revealed several significantly enriched metabolic pathways, which suggests a correlation of the molecular signature with metabolic systems and may help explain the underlying mechanisms.

Conclusions: Our study identified a novel six-gene metabolic signature for LUAD prognosis prediction. The molecular signature could reflect the dysregulated metabolic microenvironment, provide potential biomarkers for predicting prognosis, and indicate potential novel metabolic molecular-targeted therapies.

\section{Introduction}

Lung cancer is the leading cause of cancer-related deaths worldwide, accounting for nearly $20 \%$ of all cancer deaths (Bray et al., 2018). Lung adenocarcinoma (LUAD) is one of the main subtypes of lung cancer (Travis, 2020), accounting for more than $40 \%$ of lung cancer cases (Hutchinson et al., 2019), and its relative frequency is increasing (Twardella et al., 2018). Despite great improvements in the treatment of LUAD, the prognosis in patients with LUAD remains poor owing to the lack of early detection and effective individual therapies (Dolly et al., 2017). Therefore, exploring prognostic biomarkers is a critical need to help predict prognosis in LUAD and to design individual therapies. Until now, most prognostic models were based on clinical characteristics (e.g., age, sex, TNM stage, vascular tumor invasion, and organization classification) or a single molecular biomarker, such as carcinoembryonic antigen and epidermal growth factor receptor. However, these prognostic models have limited power for predicting prognosis because of the complicated molecular mechanisms of LUAD development and 
77 progression. Therefore, it is important to explore the mechanism of LUAD pathology in more

78 depth using bioinformatics to construct prognostic models that predict the patient' prognosis

79 more accurately.

80 Metabolic reprogramming is one of the hallmarks of cancer (Faubert et al., 2020), which takes

81 place from the onset and throughout the development of cancer (Chang, Fang \& Gu, 2020). It

82 plays an important role in the progression, metastasis, depressed immunity, and therapy

83 resistance of cancer (Lane et al., 2019). Metabolic reprogramming has been widely accepted as

84 the basis for the discovery of novel tumor biomarkers. Satriano et al. (2019) observed that

85 metabolic rearrangement played an important role in predicting the prognosis in patients with

86 primary liver cancers. Chen et al. (2019) revealed that reprogrammed tumor glucose metabolism

87 could promote cancer stemness and result in poor prognosis in breast cancer patients. There are

88 hundreds of metabolic genes that consistently have an altered expression in LUAD

89 (Asavasupreechar et al., 2019; Vanhove et al., 2019); however, their roles and mechanisms of

90 action remain unclear. This study investigated the role of abnormal metabolism in predicting the 91 prognosis in patients with LUAD.

92 With the development of genome sequencing and bioinformatics, new data have emerged.

93 Prognosis-related gene signatures that were constructed using these new tools have made great

94 contributions to tumor prognosis prediction. This study aimed to use bioinformatic methods to

95 establish a prognostic metabolic-gene molecular model that can predict prognosis in patients

96 with LUAD. This model could potentially guide personalized therapy for such patients.

97

\section{Materials \& Methods}

99 Data expression datasets

100 The transcriptome expression profiles and corresponding clinical information for LUAD were 101 downloaded from the Cancer Genome Atlas (TCGA; http://portal.gdc.cancer.gov/) and Gene 102 Expression Omnibus (GEO; http://www.ncbi.nlm.nih.gov/geo/) databases. From the TCGA, 103 gene expression data were of the HTSeq-FPKM type, obtained from 497 LUAD and 54 non104 tumor samples. From the GEO, the GSE68465 dataset included 443 LUAD and 19 non-tumor 105 samples, using the GPL96 platform (Affymetrix Human Genome U133A Array). The metabolic 106 genes in the Kyoto Encyclopedia of Genes and Genomes (KEGG) pathway were extracted from 107 Gene Set Enrichment Analysis (GSEA) (http://software.broadinstitute.org/gsea/index.jsp/), and 108 the overlapping metabolism-related genes were identified from TCGA and GSE68465

109 (Possemato et al., 2011; Zhu et al., 2020).

110

111 Construction and validation of the prognostic metabolic gene signature

112 The clinical cases from the TCGA database were used to assess the prognostic associations of

113 the metabolic genes with clinical outcomes. The differentially expressed genes (DEGs) between 114 LUAD and paired non-tumor samples were obtained by the Wilcoxon rank sum test using the $\mathrm{R}$ 115 package called "limma", and the adjusted $P$-value $<0.05$ and absolute $\log 2$ fold change (FC) $>1$ 116 were considered as the selection criterion. Univariate Cox regression analysis was used to 
117 identify prognosis-related metabolic genes, and adjusted $P$-values $<0.001$ were considered

118 statistically significant. The lasso penalty for Cox proportional hazards model (1,000 iterations)

119 was used to construct the prognostic gene-expression signature utilizing an R package called

120 "glmnet." The prognostic gene-expression signature was designed using a risk scoring method

121 with the following formula:

122

$$
\text { Risk score }=\sum_{i}^{n}\left(x_{i} * \beta_{i}\right)
$$

123

124

125

126

127

128

129

130

131

132

133

134

135

136

137

138

139

140

141

142

143

144

145

146

147

148

149

150

151

152

153

154

155

where $x_{i}$ indicates the expression of gene $i$ and $\beta_{i}$ indicates the coefficient of gene $i$ generated from the Cox multivariate regression.

The R package "survminer" was used to explore the cutoff point of the risk score, which divided patients into high- and low-risk groups. The R package "survival" was used to draw the KaplanMeier survival curves to demonstrate the overall survival (OS) in the high- and low-risk groups. The R package "survival ROC" was used to evaluate the prognostic value of the gene-expression signature.

\section{Independence of the prognostic gene signature from other clinical characteristics}

To determine whether the predictive power of the prognostic gene-expression signature could be independent from other clinicopathological variables in patients with LUAD (including age, sex, TNM stage, $\mathrm{T}$ stage, $\mathrm{N}$ stage, and $\mathrm{M}$ stage), univariate and multivariate Cox regression analyses were performed. The hazard ratio (HR), 95\% confidence intervals (Cis), and $P$-values were calculated.

\section{Construction and validation of a predictive nomogram}

The nomogram was constructed using all the independent prognostic factors of the Cox regression analyses using R package "rms." Validation of the nomogram was assessed by discrimination and calibration using the concordance index (C-index) by Harrell et al. (1996) (bootstraps with 1,000 resamples) and the calibration plot, respectively.

\section{External validation of the prognostic metabolic gene signature}

To verify the prognostic metabolic-gene molecular signature in the GEO dataset, the risk score of patients was calculated directly with the gene-expression signature constructed from the TCGA dataset for further analysis. The receiver operating characteristic (ROC) and KaplanMeier analyses were performed identically with the gene signature in the TCGA dataset. The mRNA expression levels of the signature genes were analyzed further using online databases (the Oncomine database [http://www.oncomine.org/] and TIMER database

[http://cistrome.shinyapps.io/timer/]). The protein expression levels associated with the signature genes were validated using the Human Protein Atlas database (http://www.proteinatlas.org/). The known genetic alterations of the signature genes were investigated using cBioPortal for Cancer Genomics (http://www.cbioportal.org/). 
156

157

158

159

160

161

162

163

164

165

166

167

168

169

170

171

172

173

174

175

176

177

178

179

180

181

182

183

184

185

186

187

188

189

190

191

192

193

194

195

\section{Gene Set Enrichment Analysis}

Enrichment analysis of the KEGG pathways of the signature genes was performed using GSEA on the TCGA dataset. The nominal (NOM) $P$-value $<0.05$ and the False Discovery Rate (FDR) $q$-value $<0.25$ indicated statistical significance.

\section{Statistical analysis}

All analyses were performed using R software v3.6.3 (R Foundation for Statistical Computing, Vienna, Austria). Two-tailed $P$-values $<0.05$ were considered statistically significant.

\section{Results}

\section{Clinical characteristics}

The TCGA dataset included 486 patients with LUAD (Table S1). The GEO dataset included 443 patients with LUAD (Table S1). Patients with a survival time of less than 30 days were omitted. For the study, 454 and 439 patients remained in the TCGA and GEO datasets, respectively. The detailed clinical characteristics of all patients are listed in Table $\mathbf{1 .}$

\section{Building and validation of the prognostic metabolic gene signature}

To clarify our study design, a flow chart of the analysis procedure is presented in Fig. 1. A list of 994 genes in the KEGG pathway was identified from GSEA (Table S2), and 633 overlapping metabolism-related genes were abstracted from TCGA and GSE68465 (Table S3). The 96 DEGs (72 up-regulated genes and 24 download genes) between LUAD and paired non-tumor samples were identified from the further analysis (Fig. 2; Table S4). Seven significant genes associated with OS were identified using univariate analysis (Table S4). Furthermore, six genes were selected to build the prognostic model using a lasso-penalized Cox analysis (Table 2). The six genes were phosphofructokinase platelet $(P F K P)$, pyruvate kinase muscle ( $P K M)$, triosephosphate isomerase 1 (TPII), lactate dehydrogenase A ( $L D H A)$, prostaglandin E synthase $($ PTGES $)$, and thymidylate synthase $($ TYMS $)$. Risk score $=(0.00005 \times P F K P$ mRNA level $)+$ $(0.00173 \times P K M$ mRNA level $)+(0.00038 \times$ TPIl mRNA level $)+(0.00379 \times L D H A$ mRNA level $)+(0.00292 \times$ PTGES mRNA level $)+(0.02490 \times$ TYMS mRNA level $)$.

The 445 patients with LUAD were divided into the high-risk or low-risk group based on the median risk score of 0.861 in the TCGA dataset. Patients in the high-risk group had significantly poorer OS than those in the low-risk group $(P<0.001$; Fig. 3A). The distribution of the risk score and survival status of the patients is presented in Fig. 3C, which showed a higher mortality in the high-risk group than in the low-risk group. The expression of the six prognostic genes is shown in the heatmap. All the six genes had a significant positive correlation with the high-risk group (Fig. 3E). The area under the curve (AUC) of the time-dependent ROC curve was used to identify the prognostic ability of the six-gene molecular signature. The AUCs of the six-gene signature model were $0.693,0.655$, and 0.565 for the 1-, 3-, and 5-year OS, respectively, suggesting that the prediction model had a good performance in predicting the OS in patients with LUAD (Fig. 3G). 
196

197

198

199

200

201

202

203

204

205

206

207

208

209

210

211

212

213

214

215

216

217

218

219

220

221

222

223

224

225

226

227

228

229

230

231

232

233

234

The prognostic model was validated in the GSE68465 dataset. The 439 patients with LUAD were divided into the high-risk or low-risk group based on the median risk score of 0.861 .

Patients in the high-risk group had a poor OS compared with those in the low-risk group $(P<$ 0.001; Fig. 3B). The distribution of the risk score and survival status showed a higher mortality in the high-risk group than in the low-risk group (Fig. 3D). The expression heatmap of the six prognostic genes showed that all the six genes had a significant positive correlation with the high-risk group (Fig. 3F). The AUCs of the six-gene signature model were 0.728, 0.654, and 0.618 for the 1-, 3-, and 5-year OS, respectively (Fig. 3H). Taken together, these results suggested that the prognostic model had a high sensitivity and specificity in predicting the OS in patients with LUAD.

\section{The prognostic gene signature was independent from other clinicopathological factors}

Univariate and multivariate Cox regression analyses were conducted to assess the independent predictive value of the six-gene prognostic signature. In the TCGA dataset, univariate Cox regression analysis demonstrated that the prognostic model (HR: 2.845, $P<0.001$ ), TNM stage (HR: $1.666, P<0.001$ ), T stage (HR: 1.605, $P<0.001$ ), and N stage (HR: $1.806, P<0.001$ ) had a prognostic value for OS (Fig. 4A). Multivariate Cox regression analysis demonstrated that the only prognostic model (HR: 2.448, $P<0.001$ ) and TNM stage (HR: 1.950, $P<0.01$ ) were independent prognostic factors for OS (Fig. 4A). In the GSE68465 dataset, the prognostic model, $\mathrm{T}$ stage, $\mathrm{N}$ stage, and age had a prognostic value in the univariate and multivariate Cox regression analyses (Fig. 4B). Gender was the only independent prognostic factor for OS in the univariate Cox regression analysis (Fig. 4B).

In addition, the time-dependent ROC curve was used to identify the predictive ability of the prognostic model compared with the other clinicopathological characteristics. In the TCGA dataset, the AUCs of the prognostic model were 0.693, 0.655, and 0.565 for the 1-, 3-, and 5-year OS, respectively, which were higher than most of the other clinicopathological characteristics including age $(0.498,0.511,0.485)$, gender $(0.579,0.485,0.451)$, T stage $(0.673,0.613,0.608)$, $\mathrm{N}$ stage $(0.685,0.666,0.628)$, and $\mathrm{M}$ stage $(0.508,0.527,0.530)$ (Fig. 5A). Furthermore, in the GSE68465 dataset, the AUCs of the prognostic model were 0.728, 0.654, and 0.618 for the 1-, 3, and 5-year OS, respectively, which were higher than most of the other clinicopathological characteristics including age $(0.593,0.568,0.581)$, gender $(0.539,0.549,0.547)$, grade $(0.580$, 0.571, 0.548), T stage $(0.647,0.606,0.606)$, and $N$ stage $(0.690,0.680,0.655)$ (Fig. 5B). The prognostic model had a larger AUC value compared with other clinicopathological characteristics. These results indicated that the model was an excellent prognostic model for LAUD patients, especially for the 1- and 3-year OS.

These results suggested that our prognostic model could be an independent predictor of prognosis in patients with LAUD.

\section{Building and validating a predictive nomogram}


235 A nomogram was built to predict the survival probability in patients with LAUD in the TCGA 236 dataset. The nomogram was constructed using four prognostic factors (the TNM stage, T stage, 237 N stage, and prognostic model; Fig. 6A). The $\mathrm{C}$-index was calculated to evaluate the predictive 238 ability of the nomogram for OS. The C-index for the nomogram was 0.754 (95\% CI: $0.561-$ 239 0.947). Calibration plots indicated that the nomogram had a good accuracy in predicting the 1240 and 3-year OS (Fig. 6B).

241 To predict the survival probability more accurately, the combined prognostic model was built

242 based on the nomogram. The combined prognostic model consisted of the TNM stage, T stage, N 243 stage, and prognostic model. A time-dependent ROC curve was used to identify the predictive 244 ability of the combined prognostic model. The AUCs of the combined prognostic models were $2450.782,0.717$, and 0.688 for the 1-, 3-, and 5-year OS, respectively, which were higher than other 246 clinical models including the TNM stage model $(0.732,0.687,0.681)$, T stage model $(0.671$, $2470.612,0.613)$, $\mathrm{N}$ stage model $(0.686,0.661,0.648)$, and the prognostic model $(0.692,0.634$, 248 0.576). The combined model had the largest AUC value compared with other factors, which 249 indicated that the combined model had a good predictive accuracy for survival. These results 250 suggested that the predictive ability of the combined model built with the nomograms is better 251

252

253

254

255

256

257

258

259

260

261

262

263

264

265

266

267

268

269

270

271

272

273 than other models, especially for predicting 1- and 3-year survival (Fig. 6C).

\section{Gene Set Enrichment Analysis}

To recognize signaling pathways that are differentially activated in LUAD, a GSEA was used, and a total of 49 significantly enriched KEGG pathways were found in the high-risk group and low-risk group (Table S5) of the TCGA dataset (FDR $q$-val $<0.25$, NOM $p$-val $<0.05$ ). Among them, many enriched pathways were related to metabolism and some highly dysregulated pathways including cell cycle, p53 signaling pathway, and basal transcription factors were also contained in these results (Table S5). We chose the top five significantly enriched metabolismsignaling pathways depending on the normalized enrichment score from the high-risk group or low-risk group. We found that the top five most significantly enriched metabolism-related pathways of the high-risk group were the cysteine and methionine, fructose and mannose, glyoxylate and dicarboxylate, purine, and pyrimidine pathways (Fig. 7A). The top five most significantly enriched metabolism-related pathways of the low-risk group were the alpha linolenic acid, arachidonic acid, ether lipid, glycerophospholipid, and linoleic acid pathways (Fig. 7B). Most of the metabolism-related pathways in the high-risk group mainly focused on amino acid and glycolysis metabolism, while the pathways in the low-risk group mainly focused on lipid metabolism. The results of the ten representative enriched metabolism-related KEGG pathways are given in Table 3. Furthermore, all the six metabolic genes of the prognostic model enriched these metabolism pathways significantly. LDHA enriched the cysteine and methionine pathway (Table S6); PFKP and TPI1 enriched the fructose and mannose pathway (Table S6); PKM enriched the purine pathway (Table S6); TYMS enriched the pyrimidine pathway (Table S6); and PTGES enriched the arachidonic acid pathway (Table S6). The results further 
274 elucidated the role of metabolism in LUAD and the value of the six-gene signature in predicting

275 the prognosis of LUAD.

276

277 External validation using online databases

278 To further identify the role of the six metabolic genes in LUAD, we compared the mRNA

279 expression levels of the six metabolic genes (PFKP, PKM, TPI1, LDHA, PTGES, and TYMS) in

280 the LAUD tissues with those in the normal lung tissues using data from the Oncomine database

281 (Fig. 8). Obviously, all the six genes were overexpressed in lung cancer in all the datasets from

282 the Oncomine database with the threshold of fold change $=2, P$-value $=0.001$ (Fig. 8A).

283 Furthermore, the mRNA levels of all the six genes in LUAD were significantly upregulated than

284 those in normal tissues in the combined LUAD datasets from the Oncomine database (Fig. 8B;

285 Table 4). To further validate the overexpression of the six genes in LUAD, we analyzed the

286 expression of the six genes using TIMER databases (Fig. 9). The results revealed that all the

287 mRNA expression of the six genes in LUAD were significantly higher than in normal tissues. All

288 the results from the Oncomine and TIMER databases were consistent with our results for the

289 TCGA and GEO datasets. In addition, the mRNA expression of the six genes was also higher in

290 esophageal carcinoma, head and neck squamous cell carcinoma, lung squamous cell carcinoma,

291 and stomach adenocarcinoma from the TIMER databases (Fig. 9). The protein expressions of

292 these six genes were analyzed using clinical specimens from the Human Protein Profiles (Fig.

293 10A and 10B; Table 5). The representative images of the six gene protein levels from the

294 Human Protein Profiles are shown in Fig. 10A. Compared with the expression level in normal

295 lung tissue, $\operatorname{LDHA}(100 \%, \mathrm{n}=7)$ and $\operatorname{TYMS}(80 \%, \mathrm{n}=5)$ showed a significantly higher percentage

296 of high/medium expression levels in the LAUD tissue (Fig. 10B; Table 5). PKM (50\%, $\mathrm{n}=6)$,

297 PFKP (33.33\%, n=6), and PTGES (16.67\%, n=6) showed a significant moderate percentage of

298 high/medium expression levels in the LAUD tissue (Fig. 10B; Table 5). However, TPI1 showed

299 no detected expression both in the LAUD and normal lung tissue (Fig. 10B; Table 5). The

300 genetic alterations were explored in the cBioPortal database. Amplifications and mutations were

301 the most common alterations in the six metabolic genes (Fig. 10C). The aberrant genetic

302 alterations might elucidate the overexpression of these six genes in LUAD.

303 Altogether, the correlation of the aberrant expression of these six genes with LAUD cancer

304 was further validated using multiple online databases.

305

306 Discussion

307 LUAD is the most common histological subtype of primary lung cancer. The incidence of

308 LUAD has been increasing rapidly, and mortality has not significantly decreased despite great

309 improvements in research and treatment. Therefore, exploring the molecular mechanisms of

310 LUAD progression and constructing a valid and accurate molecule-based tool for evaluating the

311 prognosis in patients is urgently needed. This could help design more efficient therapeutic

312 strategies for LUAD. Metabolic reprogramming in cancers could lead to their development and

313 progression (Nwosu et al., 2017; Liu et al., 2020). Characterization of the changes in metabolic 
314

315

316

317

318

319

320

321

322

323

324

325

326

327

328

329

330

331

332

333

334

335

336

337

338

339

340

341

342

343

344

345

346

347

348

349

350

351

352

353

gene expression in LUAD would allow development of novel prognostic biomarkers. However, a single biomarker is not a robust measure for predicting patient prognosis. Thus, constructing a robust multiple-biomarker signature for predicting the prognosis in cancer patients is necessary.

We identified and designed a novel six-gene prognostic molecular signature based on the TCGA database and validated its efficiency in the GSE68465 dataset. The results indicated that the molecular signature was significantly associated with OS in patients with LUAD in the training and validation sets. These results indicate that the molecular signature has a robust prognostic value, especially for predicting short-term survival in patients with LUAD. These results also demonstrated that the prognostic signature was independent of other clinicopathological characteristics, which further supports the prognostic value of this signature.

To increase the accuracy of the prediction of prognosis, we constructed a nomogram built with the combination of genetic and clinically related variables of patients with LUAD. The nomogram included the prognostic model, TNM, T stage, and $\mathrm{N}$ stage. Its predictive accuracy was verified using calibration plots, the C-index, and the AUC, which indicated that the nomogram had a greater predictive value than the previous systems. The Gene Set Enrichment Analysis showed that many significantly enriched pathways were metabolism-related pathways. The different risk groups possessed different metabolic pathway features. The metabolismrelated pathways in the high-risk group were mainly associated with amino acid and glycolysis metabolism, while the pathways in the low-risk group were mainly associated with lipid metabolism. These results revealed that the different risk groups possessed the different metabolic features, which might provide the underlying metabolic mechanisms of promoting the prognosis of LUAD. All these results further suggest a strong association between the molecular signature and metabolic systems and might reflect the dysregulated metabolic microenvironment of cancers.

Most of the six genes in our prognostic signature are suggested to be related to cancer development. PFKP is a major isoform of cancer-specific phosphofructokinase-1, an enzyme that catalyzes the phosphorylation of fructose-6-phosphate to form fructose-1,6-bisphosphate. Recently, PFKP was noted to have an aberrant upregulation in many cancers, such as breast cancer, prostate cancer, and glioblastoma. The dynamic upregulation of $P F K P$ promotes metabolic reprogramming and cancer cell survival (Bjerre et at., 2019; Kim et al., 2017). As a key regulator enzyme in glycolysis, PFKP enriched the fructose and mannose metabolism pathway. Recent studies showed that PFKP is highly expressed in lung cancer and promotes lung cancer development via fructose and mannose metabolism (Shen et al., 2020; Wang, et al., 2015). $P K M$ is a rate-limiting enzyme in the final step of glycolysis, that is considered as one of the metabolic hallmarks of cancer (Prakasam et al., 2017). The abnormal expression of PKM promoted cancer growth, invasion, and metastasis by governing aerobic glycolysis (Prakasam et al., 2017; Zahra et al., 2020) and induced cancer treatment resistance (Calabretta et al., 2016). Furthermore, $P K M$ is overexpressed in non-small cell lung cancer (NSCLC) and involved in the development and prognosis of NSCLC (Luo et al., 2018). TPII is a crucial enzyme in carbohydrate metabolism, catalyzing the interconversion of dihydroxyacetone phosphate and d- 
354

355

356

357

358

359

360

361

362

363

364

365

366

367

368

369

370

371

372

373

374

375

376

377

378

379

380

381

382

383

384

385

386

387

388

389

390

391

392

393

glyceraldehyde-3-phosphate during glycolysis and gluconeogenesis. TPIl is abnormally expressed in different kinds of cancers, such as breast cancer, gastric cancer, and lymphoma and is associated with a poor prognosis in patients with neuroblastoma and pancreatic cancer through dysregulating glycometabolism (Ludvigsen et al., 2018; Applebaum et al., 2016; Follia et al., 2019). LDHA is an enzyme that catalyzes the interconversion of pyruvate and lactate. LDHA was enriched in cysteine and methionine metabolism, and its aberrant metabolism regulation promoted many pathological processes in tumors, such as cell proliferation, survival, invasion, metastasis, and immunity (Dorneburg et al., 2018). Overexpressed LDHA is associated with poor prognosis in many tumors, including NSCLC, breast cancer, gallbladder carcinoma, and gastrointestinal cancer (Mizuno et al., 2020; Guddeti et al., 2019). PTGES is a key enzyme in the arachidonic acid metabolism pathway. An abnormally high expression of PTGES is correlated with proliferation, invasion, and metastasis in many cancer cells (Kim et al., 2016; DelgadoGoñi et al., 2020). The dysregulated PTGES promoted tumor migration and metastasis of lung cancer cells and played an important role in lung cancer progression (Wang et al., 2019). TYMS is a rate-limiting enzyme, which plays an important role in regulating the pyrimidine metabolism signaling pathway (Yeh et al., 2017). TYMS is overexpressed frequently in different kinds of cancers, such as NSCLC, pancreatic, colorectal, and breast cancers, and it has resulted in a poor cancer prognosis and chemotherapy resistance via dysregulating pyrimidine metabolism (Troncarelli Flores et al., 2019; Wu et al., 2019). In our study, we constructed a six-gene signature for a prognostic model based on the TCGA database. This novel six-gene signature had a higher survival prediction, and the predictive ability of this signature was further validated by the GSE68465 dataset and multiple online databases. To our knowledge, the six-gene signature for prognosis prediction in LUAD has not been reported yet. Compared with the traditional prognostic models such as clinical characteristics (e.g., TNM stage, vascular tumor invasion, and organization classification) or a single molecular biomarker, a multi-gene signature can predict the prognosis more accurately and provide a clearer molecular mechanism for personalized LUAD therapy.

There are limitations in our study. First, our nomogram was not validated further in the GEO database because the GSE68465 lacked detailed TNM stage data. Thus, the nomogram should be externally validated using larger datasets from multicenter clinical trials and perspective studies. Second, functional experiments should be further performed to explore the molecular mechanisms predicted by the metabolic gene expression.

\section{Conclusions}

We concluded from our research results that the six-gene metabolic prognostic signature could accurately predict the prognosis in patients with LUAD. The molecular signature may provide potential biomarkers for metabolic therapy and prognosis prediction of LUAD.

PeerJ reviewing PDF | (2020:07:51200:1:0:NEW 10 Oct 2020) 
394

395

396

397

398

399

400

401

402

403

404

405

406

407

408

409

410

411

412

413

414

415

416

417

418

419

420

421

422

423

424

425

426

427

428

429

430

431

\section{References}

Applebaum, M. A., Jha, A. R., Kao, C., Hernandez, K. M., DeWane, G., Salwen, H. R., Chlenski, A., Dobratic, M., Mariani, C. J., Godley, L. A., Prabhakar, N., White, K., Stranger, B. E., \& Cohn, S. L. 2016. Integrative genomics reveals hypoxia inducible genes that are associated with a poor prognosis in neuroblastoma patients. Oncotarget 7(47):7681676826 DOI 10.18632/oncotarget.12713.

Asavasupreechar, T., Chan, M., Saito, R., Miki, Y., Boonyaratanakornkit, V., \& Sasano, H. 2019. Sex steroid metabolism and actions in non-small cell lung carcinoma. The Journal of steroid biochemistry and molecular biology 193:105440 DOI 10.1016/j.jsbmb.2019.105440.

Beer, D. G., Kardia, S. L., Huang, C. C., Giordano, T. J., Levin, A. M., Misek, D. E., Lin, L., Chen, G., Gharib, T. G., Thomas, D. G., Lizyness, M. L., Kuick, R., Hayasaka, S., Taylor, J. M., Iannettoni, M. D., Orringer, M. B., \& Hanash, S. 2002. Gene-expression profiles predict survival of patients with lung adenocarcinoma. Nature medicine 8(8):816-824 DOI $10.1038 / \mathrm{nm} 733$.

Bjerre, M. T., Strand, S. H., Nørgaard, M., Kristensen, H., Rasmussen, A. K., Mortensen, M. M., Fredsøe, J., Mouritzen, P., Ulhøi, B., Ørntoft, T., Borre, M., \& Sørensen, K. D. 2019. Aberrant DOCK2, GRASP, HIF3A and PKFP Hypermethylation has Potential as a Prognostic Biomarker for Prostate Cancer. International journal of molecular sciences 20(5):1173 DOI 10.3390/ijms20051173.

Bray, F., Ferlay, J., Soerjomataram, I., Siegel, R. L., Torre, L. A., \& Jemal, A. 2018. Global cancer statistics 2018: GLOBOCAN estimates of incidence and mortality worldwide for 36 cancers in 185 countries. CA: a cancer journal for clinicians 68(6):394-424 DOI 10.3322/caac. 21492.

Calabretta, S., Bielli, P., Passacantilli, I., Pilozzi, E., Fendrich, V., Capurso, G., Fave, G. D., \& Sette, C. 2016. Modulation of PKM alternative splicing by PTBP1 promotes gemcitabine resistance in pancreatic cancer cells. Oncogene 35(16):2031-2039 DOI 10.1038/onc.2015.270.

Chang, L., Fang, S., \& Gu, W. 2020. The Molecular Mechanism of Metabolic Remodeling in Lung Cancer. Journal of Cancer 11(6):1403-1411 DOI 10.7150/jca.31406.

Chen, C., Bai, L., Cao, F., Wang, S., He, H., Song, M., Chen, H., Liu, Y., Guo, J., Si, Q., Pan, Y., Zhu, R., Chuang, T. H., Xiang, R., \& Luo, Y. 2019. Targeting LIN28B reprograms tumor glucose metabolism and acidic microenvironment to suppress cancer stemness and metastasis. Oncogene 38(23):4527-4539 DOI 10.1038/s41388-019-0735-4.

Delgado-Goñi, T., Galobart, T. C., Wantuch, S., Normantaite, D., Leach, M. O., Whittaker, S. R., \& Beloueche-Babari, M. 2020. Increased inflammatory lipid metabolism and anaplerotic mitochondrial activation follow acquired resistance to vemurafenib in BRAFmutant melanoma cells. British journal of cancer 122(1):72-81 DOI 10.1038/s41416-0190628-x. 
432

433

434

435

436

437

438

439

440

441

442

443

444

445

446

447

448

449

450

451

452

453

454

455

456

457

458

459

460

461

462

463

464

465

466

467

468

469

470

Dolly, S. O., Collins, D. C., Sundar, R., Popat, S., \& Yap, T. A. 2017. Advances in the Development of Molecularly Targeted Agents in Non-Small-Cell Lung Cancer. Drugs 77(8): 813-827 DOI 10.1007/s40265-017-0732-2.

Dorneburg, C., Fischer, M., Barth, T., Mueller-Klieser, W., Hero, B., Gecht, J., Carter, D. R., de Preter, K., Mayer, B., Christner, L., Speleman, F., Marshall, G. M., Debatin, K. M., \& Beltinger, C. 2018. LDHA in Neuroblastoma Is Associated with Poor Outcome and Its Depletion Decreases Neuroblastoma Growth Independent of Aerobic Glycolysis. Clinical cancer research 24(22):5772-5783 DOI 10.1158/1078-0432.CCR-17-2578.

Exposito, F., Villalba, M., Redrado, M., de Aberasturi, A. L., Cirauqui, C., Redin, E., Guruceaga, E., de Andrea, C., Vicent, S., Ajona, D., Montuenga, L. M., Pio, R., \& Calvo, A. 2019. Targeting of TMPRSS4 sensitizes lung cancer cells to chemotherapy by impairing the proliferation machinery. Cancer letters 453: 21-33 DOI 10.1016/j.canlet.2019.03.013.

Faubert, B., Solmonson, A., \& DeBerardinis, R. J. 2020. Metabolic reprogramming and cancer progression. Science 368(6487): eaaw5473 DOI 10.1126/science.aaw5473.

Follia, L., Ferrero, G., Mandili, G., Beccuti, M., Giordano, D., Spadi, R., Satolli, M. A., Evangelista, A., Katayama, H., Hong, W., Momin, A. A., Capello, M., Hanash, S. M., Novelli, F., \& Cordero, F. 2019. Integrative Analysis of Novel Metabolic Subtypes in Pancreatic Cancer Fosters New Prognostic Biomarkers. Frontiers in oncology 9:115 DOI 10.3389/fonc.2019.00115.

Garber, M. E., Troyanskaya, O. G., Schluens, K., Petersen, S., Thaesler, Z., PacynaGengelbach, M., van de Rijn, M., Rosen, G. D., Perou, C. M., Whyte, R. I., Altman, R. B., Brown, P. O., Botstein, D., \& Petersen, I. 2001. Diversity of gene expression in adenocarcinoma of the lung. Proceedings of the National Academy of Sciences of the United States of America 98(24):13784-13789 DOI 10.1073/pnas.241500798.

Guddeti, R. K., Bali, P., Karyala, P., \& Pakala, S. B. 2019. MTA1 coregulator regulates LDHA expression and function in breast cancer. Biochemical and biophysical research communications 520(1):54-59 DOI 10.1016/j.bbrc.2019.09.078.

Hou, J., Aerts, J., den Hamer, B., van Ijcken, W., den Bakker, M., Riegman, P., van der Leest, C., van der Spek, P., Foekens, J. A., Hoogsteden, H. C., Grosveld, F., \& Philipsen, S. 2010. Gene expression-based classification of non-small cell lung carcinomas and survival prediction. PloS one 5(4):e10312 DOI 10.1371/journal.pone.0010312.

Hutchinson, B. D., Shroff, G. S., Truong, M. T., \& Ko, J. P. 2019. Spectrum of Lung Adenocarcinoma. Seminars in ultrasound, CT, and MR 40(3):255-264 DOI 10.1053/j.sult.2018.11.009.

Kim, N. H., Cha, Y. H., Lee, J., Lee, S. H., Yang, J. H., Yun, J. S., Cho, E. S., Zhang, X., Nam, M., Kim, N., Yuk, Y. S., Cha, S. Y., Lee, Y., Ryu, J. K., Park, S., Cheong, J. H., Kang, S. W., Kim, S. Y., Hwang, G. S., Yook, J. I., Kim, H. S. 2017. Snail reprograms glucose metabolism by repressing phosphofructokinase PFKP allowing cancer cell survival under metabolic stress. Nature communications 8:14374 DOI 10.1038/ncomms14374.

PeerJ reviewing PDF | (2020:07:51200:1:0:NEW 10 Oct 2020) 
471

472

473

474

475

476

477

478

479

480

481

482

483

484

485

486

487

488

489

490

491

492

493

494

495

496

497

498

499

500

501

502

503

504

505

506

507

508

509

510

Kim, S. B., Bozeman, R. G., Kaisani, A., Kim, W., Zhang, L., Richardson, J. A., Wright, W. E., \& Shay, J. W. 2016. Radiation promotes colorectal cancer initiation and progression by inducing senescence-associated inflammatory responses. Oncogene 35(26):3365-3375 DOI 10.1038/onc.2015.395.

Landi, M. T., Dracheva, T., Rotunno, M., Figueroa, J. D., Liu, H., Dasgupta, A., Mann, F. E., Fukuoka, J., Hames, M., Bergen, A. W., Murphy, S. E., Yang, P., Pesatori, A. C., Consonni, D., Bertazzi, P. A., Wacholder, S., Shih, J. H., Caporaso, N. E., \& Jen, J. 2008. Gene expression signature of cigarette smoking and its role in lung adenocarcinoma development and survival. PloS one 3(2): e1651 DOI 10.1371/journal.pone.0001651.

Lane, A. N., Higashi, R. M., \& Fan, T. W. 2019. Metabolic reprogramming in tumors: Contributions of the tumor microenvironment. Genes \& diseases 7(2):185-198 DOI 10.1016/j.gendis.2019.10.007.

Liu, G. M., Xie, W. X., Zhang, C. Y., \& Xu, J. W. 2020. Identification of a four-gene metabolic signature predicting overall survival for hepatocellular carcinoma. Journal of cellular physiology 235(2):1624-1636 DOI 10.1002/jcp.29081.

Ludvigsen, M., Bjerregård Pedersen, M., Lystlund Lauridsen, K., Svenstrup Poulsen, T., Hamilton-Dutoit, S. J., Besenbacher, S., Bendix, K., Møller, M. B., Nørgaard, P., d'Amore, F., \& Honoré, B. 2018. Proteomic profiling identifies outcome-predictive markers in patients with peripheral T-cell lymphoma, not otherwise specified. Blood advances 2(19):2533-2542 DOI 10.1182/bloodadvances.2018019893.

Luo, M., Luo, Y., Mao, N., Huang, G., Teng, C., Wang, H., Wu, J., Liao, X., \& Yang, J. 2018. Cancer-Associated Fibroblasts Accelerate Malignant Progression of Non-Small Cell Lung Cancer via Connexin 43-Formed Unidirectional Gap Junctional Intercellular Communication. Cellular physiology and biochemistry 51(1):315-336 DOI 10.1159/000495232.

Mizuno, Y., Hattori, K., Taniguchi, K., Tanaka, K., Uchiyama, K., \& Hirose, Y. 2020. Intratumoral heterogeneity of glutaminase and lactate dehydrogenase A protein expression in colorectal cancer. Oncology letters 19(4):2934-2942 DOI 10.3892/ol.2020.11390.

Nwosu, Z. C., Megger, D. A., Hammad, S., Sitek, B., Roessler, S., Ebert, M. P., Meyer, C., \& Dooley, S. 2017. Identification of the Consistently Altered Metabolic Targets in Human Hepatocellular Carcinoma. Cellular and molecular gastroenterology and hepatology 4(2):303-323.e1 DOI 10.1016/j.jcmgh.2017.05.004.

Possemato, R., Marks, K. M., Shaul, Y. D., Pacold, M. E., Kim, D., Birsoy, K., Sethumadhavan, S., Woo, H. K., Jang, H. G., Jha, A. K., Chen, W. W., Barrett, F. G., Stransky, N., Tsun, Z. Y., Cowley, G. S., Barretina, J., Kalaany, N. Y., Hsu, P. P., Ottina, K., Chan, A. M., ... Sabatini, D. M. 2011. Functional genomics reveal that the serine synthesis pathway is essential in breast cancer. Nature 476(7360):346-350 DOI 10.1038/nature10350.

Prakasam, G., Singh, R. K., Iqbal, M. A., Saini, S. K., Tiku, A. B., \& Bamezai, R. 2017. Pyruvate kinase M knockdown-induced signaling via AMP-activated protein kinase promotes 
511

512

513

514

515

516

517

518

519

520

521

522

523

524

525

526

527

528

529

530

531

532

533

534

535

536

537

538

539

540

541

542

543

544

545

546

547

548

549

550

mitochondrial biogenesis, autophagy, and cancer cell survival. The Journal of biological chemistry 292(37):15561-15576 DOI 10.1074/jbc.M117.791343.

Satriano, L., Lewinska, M., Rodrigues, P. M., Banales, J. M., \& Andersen, J. B. 2019. Metabolic rearrangements in primary liver cancers: cause and consequences. Nature reviews. Gastroenterology \& hepatology 16(12):748-766 DOI 10.1038/s41575-019-0217-8.

Selamat, S. A., Chung, B. S., Girard, L., Zhang, W., Zhang, Y., Campan, M., Siegmund, K. D., Koss, M. N., Hagen, J. A., Lam, W. L., Lam, S., Gazdar, A. F., \& Laird-Offringa, I. A. 2012. Genome-scale analysis of DNA methylation in lung adenocarcinoma and integration with mRNA expression. Genome research 22(7):1197-1211 DOI 10.1101/gr.132662.111.

Shen, J., Jin, Z., Lv, H., Jin, K., Jonas, K., Zhu, C., \& Chen, B. 2020. PFKP is highly expressed in lung cancer and regulates glucose metabolism. Cellular oncology (Dordrecht) 43(4):617-629 DOI 10.1007/s13402-020-00508-6.

Stearman, R. S., Dwyer-Nield, L., Zerbe, L., Blaine, S. A., Chan, Z., Bunn, P. A., Jr, Johnson, G. L., Hirsch, F. R., Merrick, D. T., Franklin, W. A., Baron, A. E., Keith, R. L., Nemenoff, R. A., Malkinson, A. M., \& Geraci, M. W. 2005. Analysis of orthologous gene expression between human pulmonary adenocarcinoma and a carcinogen-induced murine model. The American journal of pathology 167(6):1763-1775 DOI 10.1016/S00029440(10)61257-6.

Su, L. J., Chang, C. W., Wu, Y. C., Chen, K. C., Lin, C. J., Liang, S. C., Lin, C. H., WhangPeng, J., Hsu, S. L., Chen, C. H., \& Huang, C. Y. 2007. Selection of DDX5 as a novel internal control for Q-RT-PCR from microarray data using a block bootstrap re-sampling scheme. BMC genomics 8:140 DOI 10.1186/1471-2164-8-140.

Travis W. D. 2020. Lung Cancer Pathology: Current Concepts. Clinics in chest medicine 41(1):67-85 DOI 10.1016/j.ccm.2019.11.001.

Troncarelli Flores, B. C., Souza E Silva, V., Ali Abdallah, E., Mello, C., Gobo Silva, M. L., Gomes Mendes, G., Camila Braun, A., Aguiar Junior, S., \& Thomé Domingos Chinen, L. 2019. Molecular and Kinetic Analyses of Circulating Tumor Cells as Predictive Markers of Treatment Response in Locally Advanced Rectal Cancer Patients. Cells 8(7):641 DOI 10.3390/cells8070641.

Twardella, D., Geiss, K., Radespiel-Tröger, M., Benner, A., Ficker, J. H., \& Meyer, M. 2018. Trends der Lungenkrebsinzidenz nach histologischem Subtyp bei Männern und Frauen in Deutschland : Analyse von Krebsregisterdaten unter Einsatz von multipler Imputation [Trends in incidence of lung cancer according to histological subtype among men and women in Germany : Analysis of cancer registry data with the application of multiple imputation techniques]. Bundesgesundheitsblatt, Gesundheitsforschung, Gesundheitsschutz 61(1):20-31 DOI 10.1007/s00103-017-2659-x.

Vanhove, K., Graulus, G. J., Mesotten, L., Thomeer, M., Derveaux, E., Noben, J. P., Guedens, W., \& Adriaensens, P. 2019. The Metabolic Landscape of Lung Cancer: New Insights in a Disturbed Glucose Metabolism. Frontiers in oncology 9:1215 DOI 10.3389/fonc.2019.01215.

Peer] reviewing PDF | (2020:07:51200:1:0:NEW 10 Oct 2020) 
551

552

553

554

555

556

557

558

559

560

561

562

563

564

565

566

567

568

569

570

571

572

573

574

575

576

577

Wang, T., Jing, B., Sun, B., Liao, Y., Song, H., Xu, D., Guo, W., Li, K., Hu, M., Liu, S., Ling, J., Kuang, Y., Feng, Y., Zhou, B. P., \& Deng, J. 2019. Stabilization of PTGES by deubiquitinase USP9X promotes metastatic features of lung cancer via PGE2 signaling. American journal of cancer research 9(6):1145-1160.

Wang, Y., Mei, Q., Ai, Y. Q., Li, R. Q., Chang, L., Li, Y. F., Xia, Y. X., Li, W. H., \& Chen, Y. 2015. Identification of lung cancer oncogenes based on the mRNA expression and single nucleotide polymorphism profile data. Neoplasma 62(6):966-973 DOI 10.4149/neo_2015_117.

Wu, Q., Zhang, B., Sun, Y., Xu, R., Hu, X., Ren, S., Ma, Q., Chen, C., Shu, J., Qi, F., He, T., Wang, W., \& Wang, Z. 2019. Identification of novel biomarkers and candidate small molecule drugs in non-small-cell lung cancer by integrated microarray analysis. OncoTargets and therapy 12:3545-3563 DOI 10.2147/OTT.S198621.

Yamagata, N., Shyr, Y., Yanagisawa, K., Edgerton, M., Dang, T. P., Gonzalez, A., Nadaf, S., Larsen, P., Roberts, J. R., Nesbitt, J. C., Jensen, R., Levy, S., Moore, J. H., Minna, J. D., \& Carbone, D. P. 2003. A training-testing approach to the molecular classification of resected non-small cell lung cancer. Clinical cancer research: an official journal of the American Association for Cancer Research 9(13):4695-4704.

Yeh, H. W., Lee, S. S., Chang, C. Y., Hu, C. M., \& Jou, Y. S. 2017. Pyrimidine metabolic rate limiting enzymes in poorly-differentiated hepatocellular carcinoma are signature genes of cancer stemness and associated with poor prognosis. Oncotarget 8(44): $77734-77751$ DOI 10.18632/oncotarget.20774.

Zahra, K., Dey, T., Ashish, Mishra, S. P., \& Pandey, U. 2020. Pyruvate Kinase M2 and Cancer: The Role of PKM2 in Promoting Tumorigenesis. Frontiers in oncology 10:159 DOI 10.3389/fonc.2020.00159.

Zhu, Z., Li, L., Xu, J., Ye, W., Chen, B., Zeng, J., \& Huang, Z. 2020. Comprehensive analysis reveals a metabolic ten-gene signature in hepatocellular carcinoma. PeerJ 8: e9201 DOI 10.7717/peerj.9201. 


\section{Figure 1}

Overall flowchart of steps used in the construction of the prognostic metabolic gene signature.

The TCGA dataset was utilized to construct the prognostic metabolic gene signature. The TCGA clinical information, GSE68465 dataset and online databases from international platforms were further utilized to validate the prognostic model. TCGA, The Cancer Genome Atlas; OS, overall survival; ROC, the receiver operating characteristic.

Platform

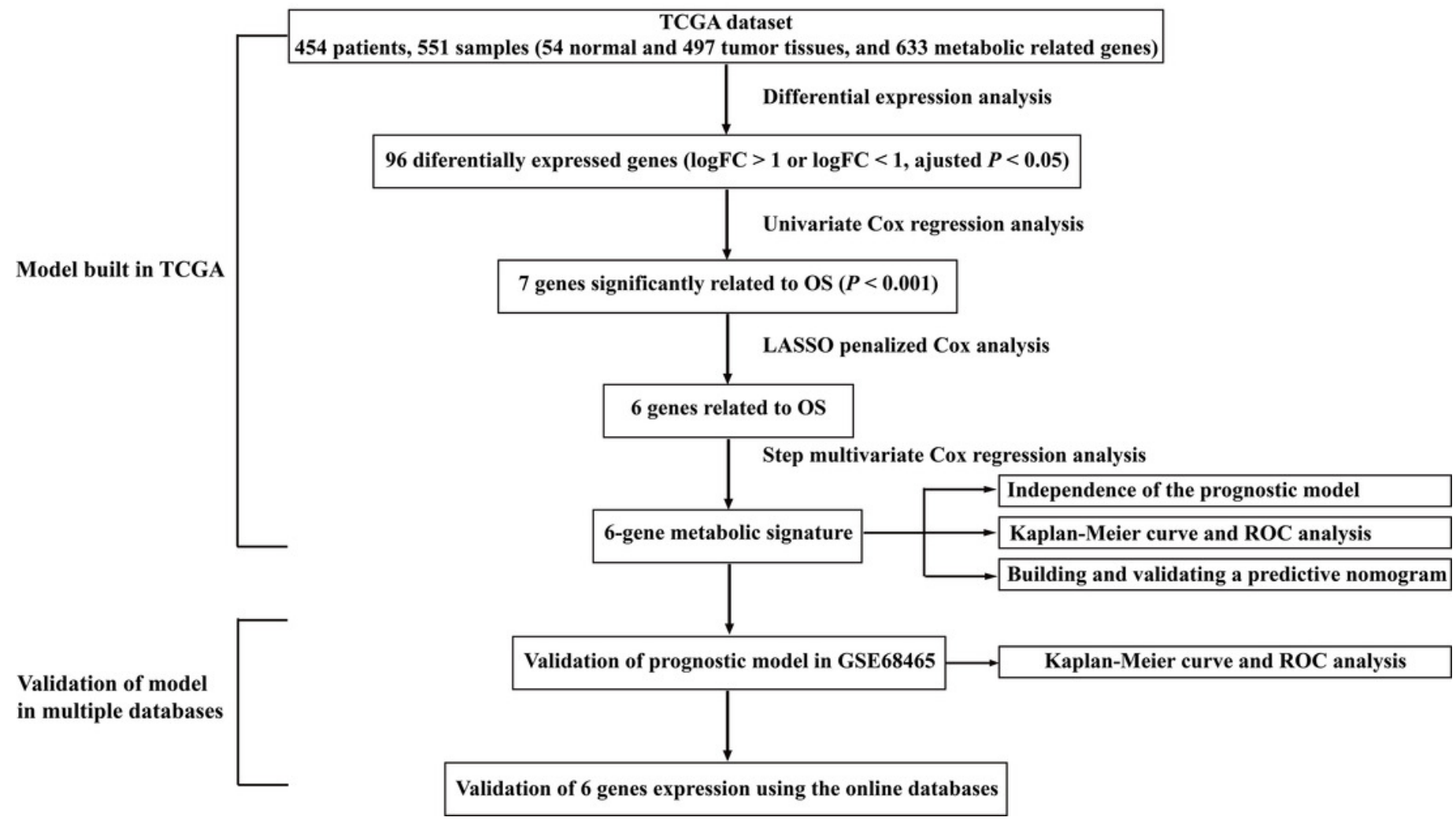




\section{Figure 2}

Heatmap and Volcano plot of metabolism-related DEGs.

(A) The heatmap of metabolism-related DEGs. The red color represented high expression genes, the green color represented low expression genes, and the black color represented the expression genes with no significant difference (FDR $<0.05$, absolute log $F C>1$ ). (B) Volcano plot of metabolism-related DEGs. The red, green and black dots represented the high expression genes, low expression genes, and the expression genes with no significant difference (FDR $<0.05$, absolute $\log F C>1$ ). DEGs, differentially expressed genes; FDR, false discovery rate.

A

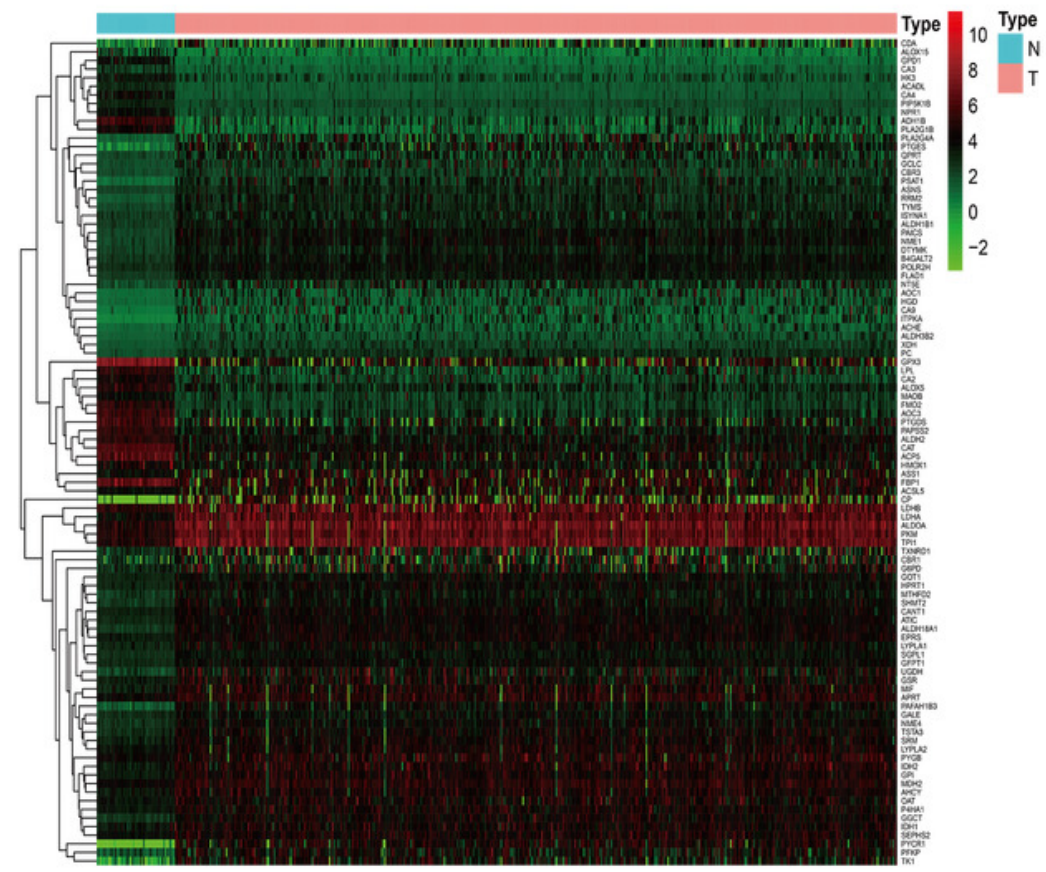

B

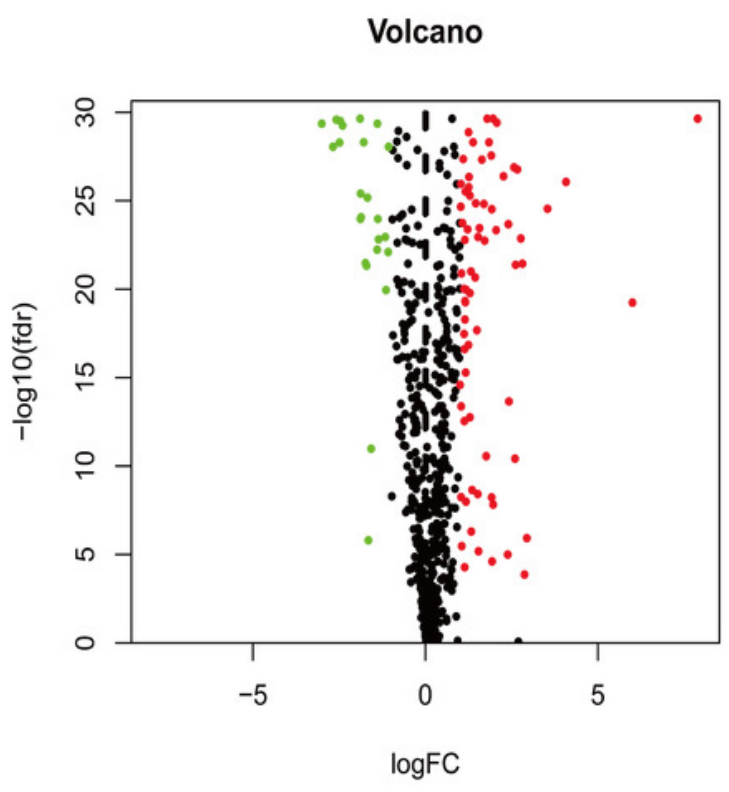




\section{Figure 3}

Identification of the prognostic model in lung adenocarcinoma.

(A, B) Kaplan-Meier curves of overall survival of the high-risk and low-risk groups stratified by the six-gene signature- based risk score in the TCGA or GEO dataset. (C, D) Risk score distribution, survival status distribution in the TCGA or GEO dataset. (E, F) The expression heatmap of the six prognostic genes in the TCGA or GEO dataset. $(G, H)$ Time-dependent ROC curves of the six-gene signature in the TCGA or GEO dataset. TCGA, The Cancer Genome Atlas; GEO, Gene Expression Omnibus; ROC, receiver operating characteristic. 
A

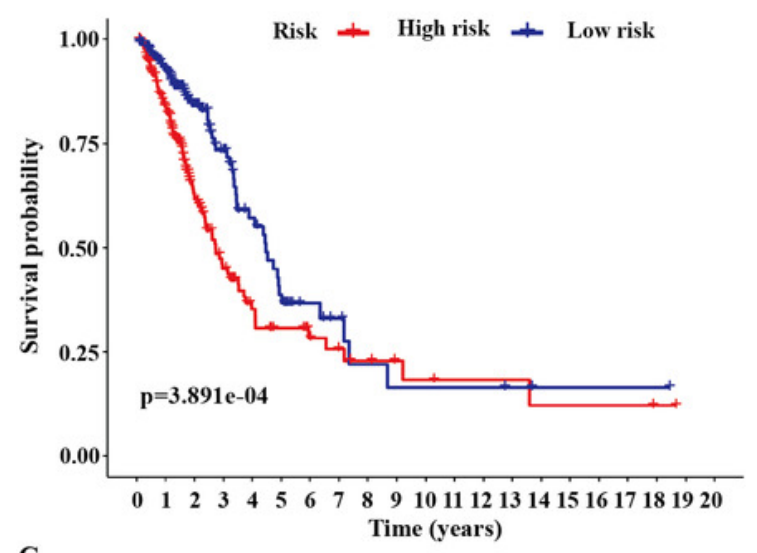

C
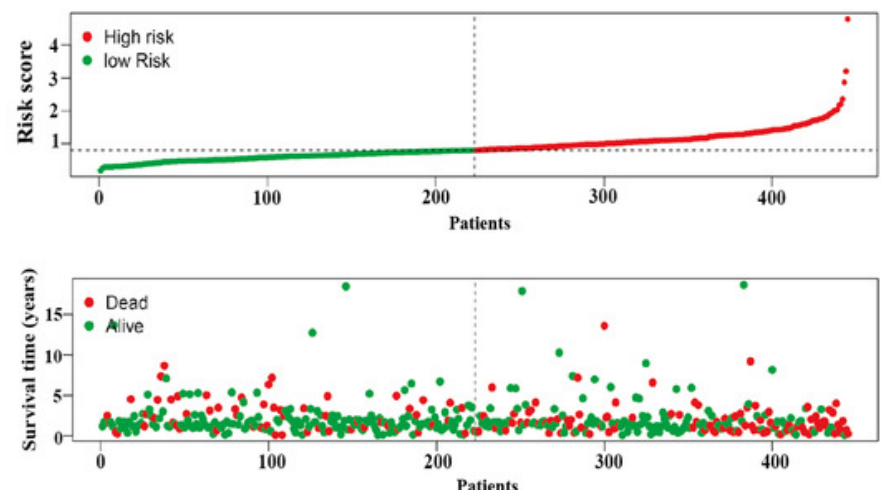

E

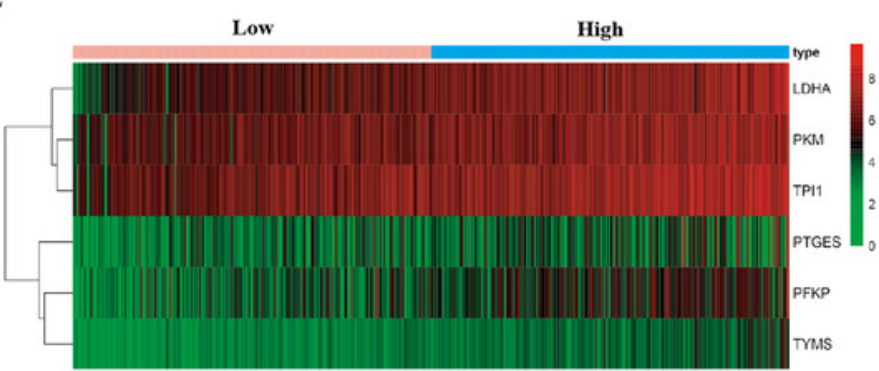

G

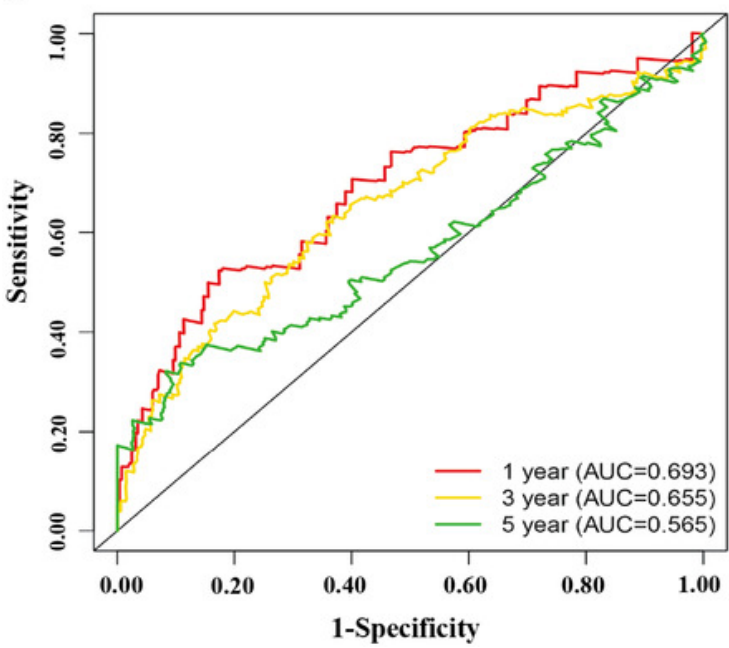

B

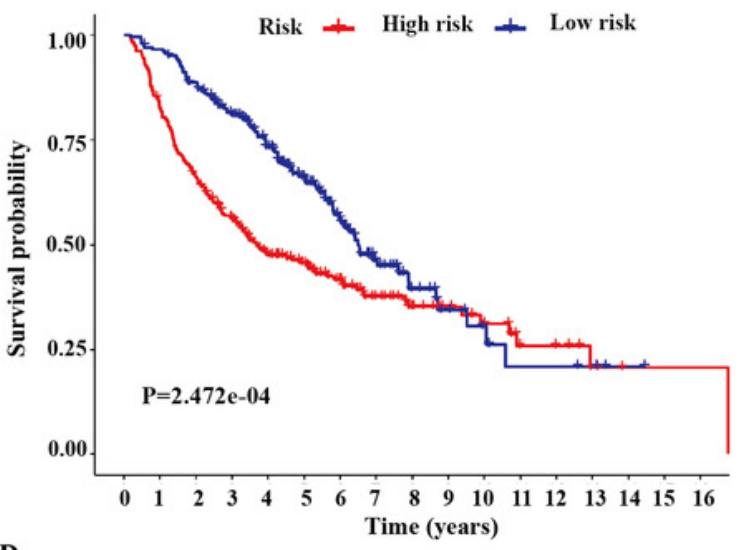

D
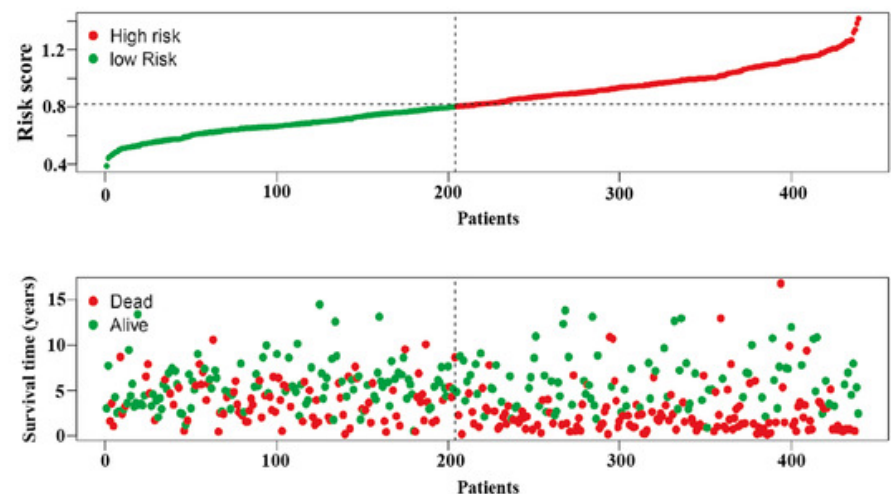

F

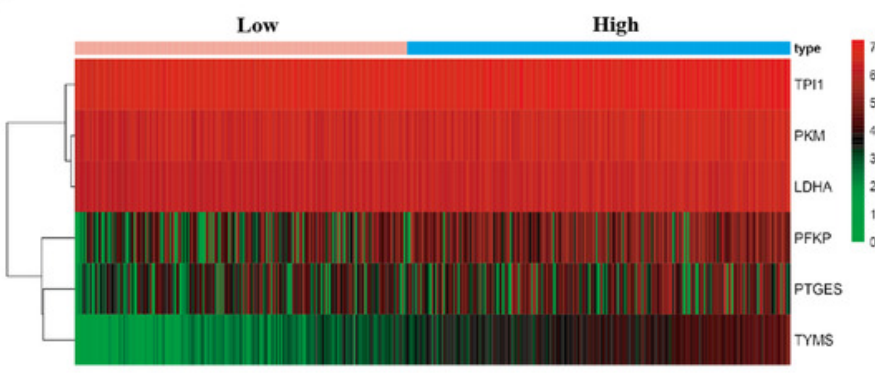

H

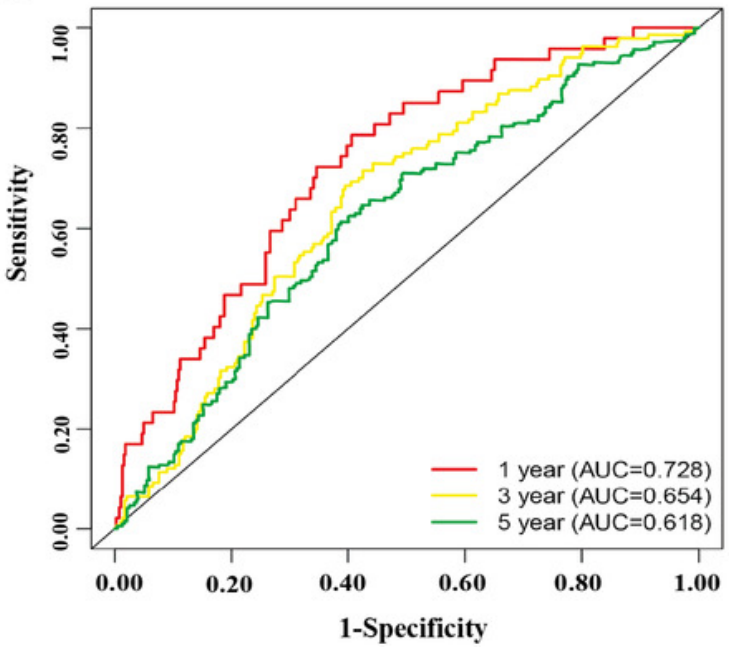


Figure 4

Cox regression analysis of the associations between the prognostic model and clinicopathological characteristics with overall survival in LAUD.

Univariate and multivariate Cox regression analyses in the TCGA dataset (A) and GEO dataset (B). LUAD, lung adenocarcinoma; TCGA, The Cancer Genome Atlas; GEO, Gene Expression Omnibus.

A Univariate Analysis

Characeristics P value Harzard Ratio $(95 \%$ CI)

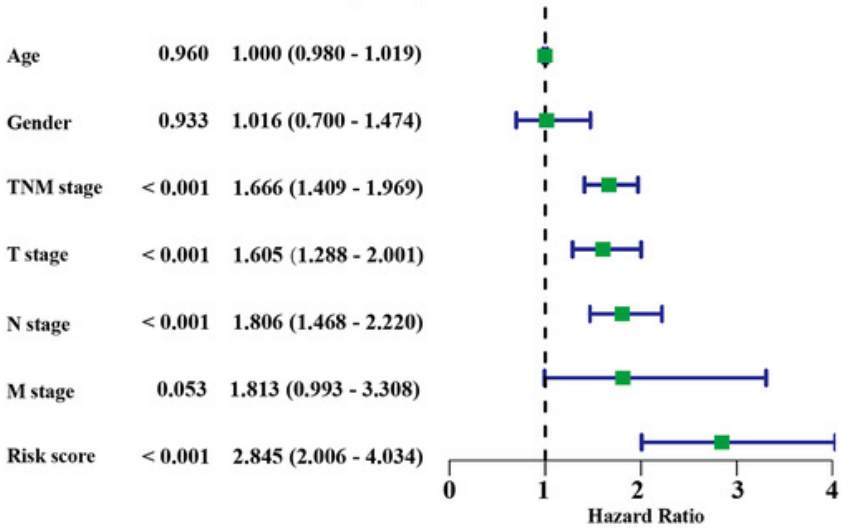

B

Univariate Analysis

Characeristics P value Harzard Ratio (95\% CI)

Age $\quad<0.001 \quad 1.026(1.012-1.039)$

Gender $\quad 0.007 \quad 1.430(1.101-1.858)$

Grade $\quad 0.177 \quad 1.145(0.941-1.394)$

T stage $\quad<0.001 \quad 1.654(1.375-1.988)$

N stage $\quad<0.001 \quad 2.021(1.715-2.381)$

Risk score

$<0.001 \quad 3.502(1.814-6.761)$

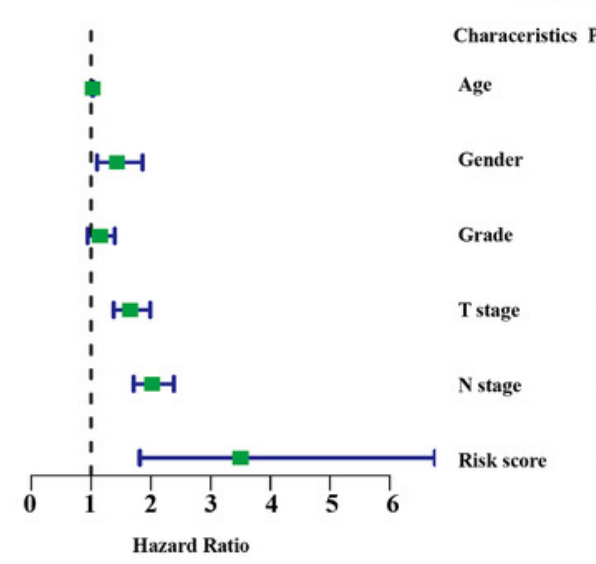

Multivariate Analysis

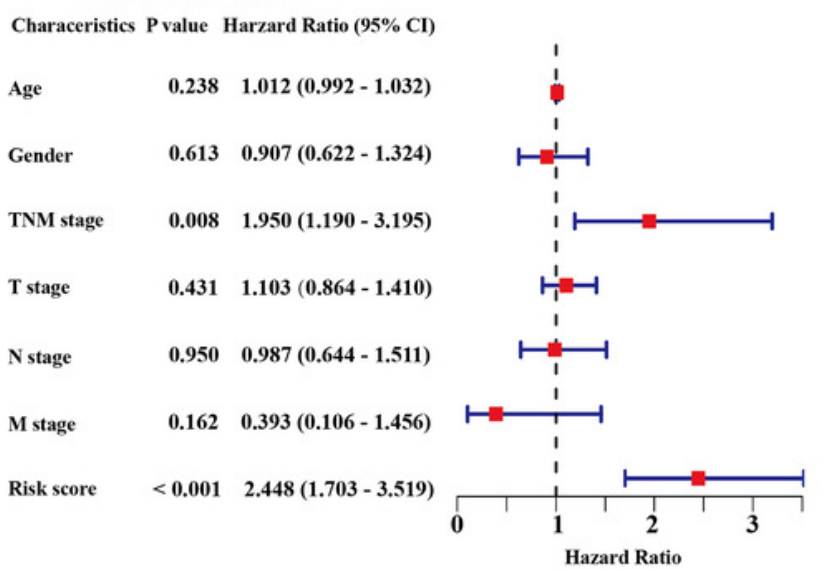

$<0.0011 .034(1.020-1.049)$

$0.150 \quad 1.216(0.932-1.586)$

$0.1110 .823(0.647-1.046)$

$<0.001 \quad 1.401(1.153-1.701)$

$<0.001 \quad 2.043(1.728-2.414)$

$<0.001 \quad 5.140(2.261-11.683)$

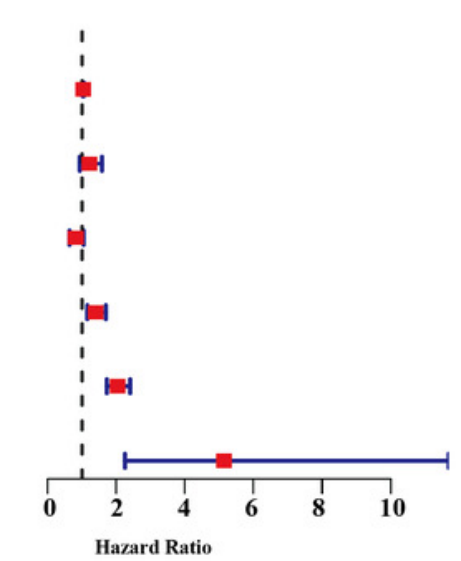


Figure 5

The time-dependent receiver operating characteristic (ROC) analysis for the prognostic model and clinicopathological characteristics in LAUD.

(A) The time-dependent ROC curves of risk score, age, gender, TNM stage, T stage, N stage, and M stage in the TCGA dataset. (B) The time-dependent ROC curves of risk score, age, gender, grade, T stage, and N stage in the GEO dataset. LUAD, lung adenocarcinoma.

A

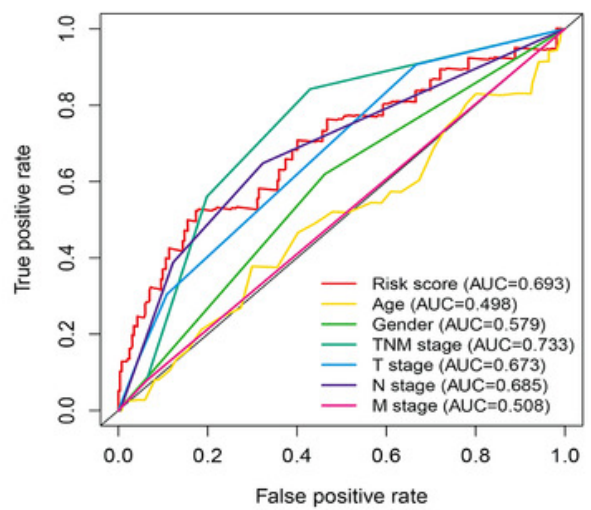

B

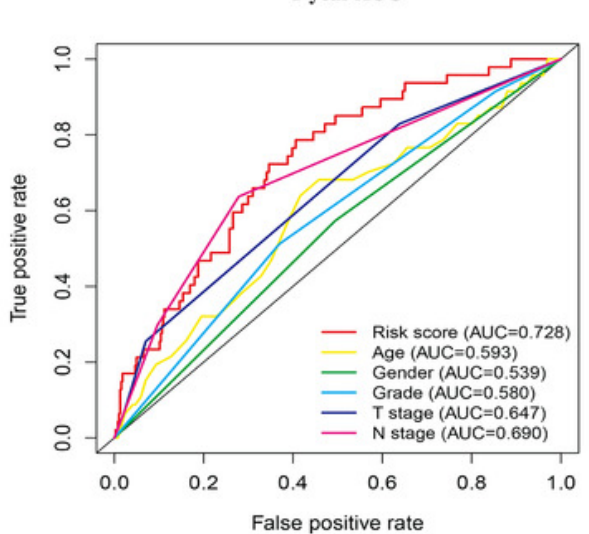

3-year AUC

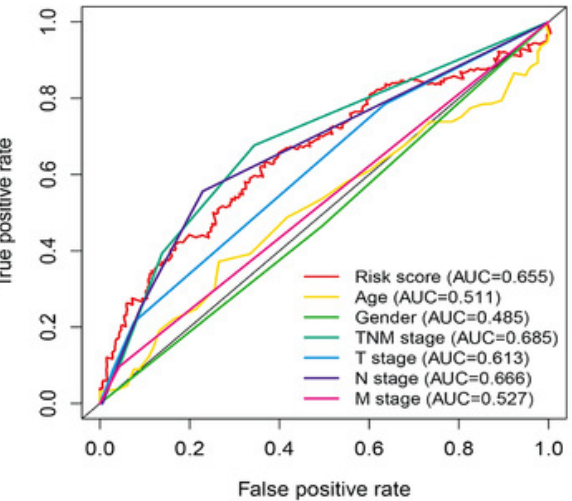

3-year AUC

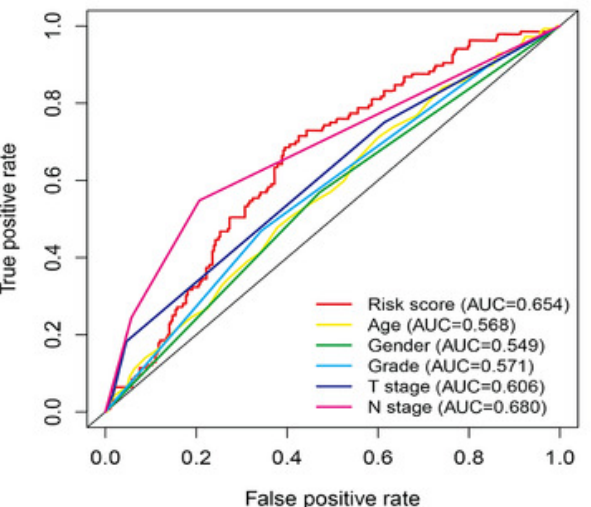

5-year AUC

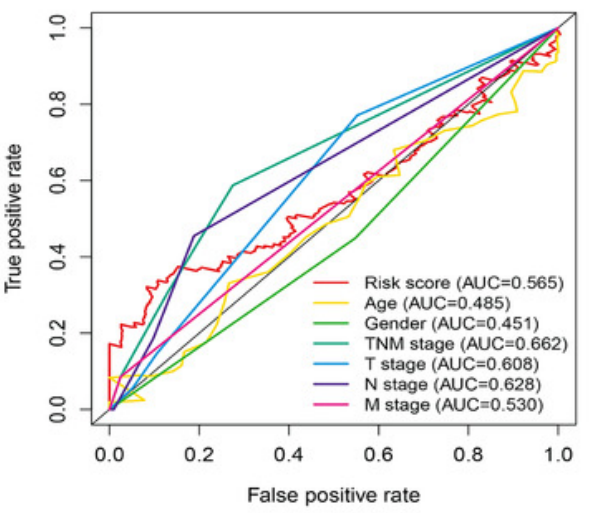

5-year AUC

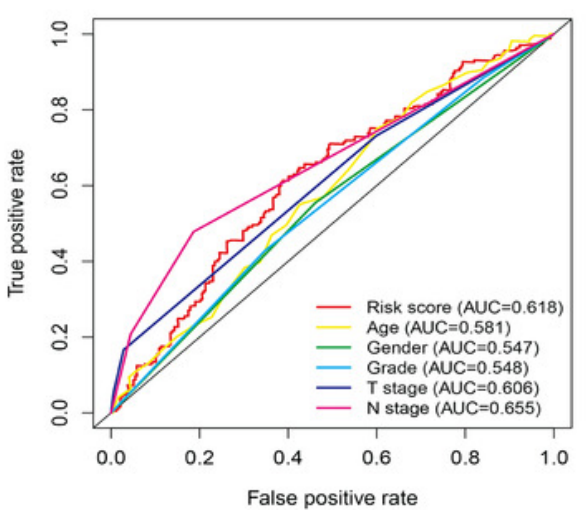




\section{Figure 6}

Construction and validation of a nomogram for survival prediction in LUAD from the TCGA dataset.

(A) The nomogram was built in the TCGA dataset. (B) Calibration plots revealed the nomogram-predicted survival probabilities. (C) The time-dependent ROC analysis evaluated the accuracy of the nomogram. TCGA, The Cancer Genome Atlas; ROC, receiver operating characteristic; LUAD, lung adenocarcinoma. 
A

Points

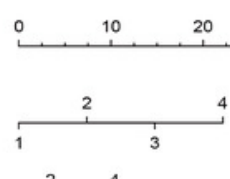

TNM stage

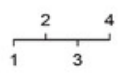

N stage

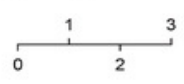

Riskscore

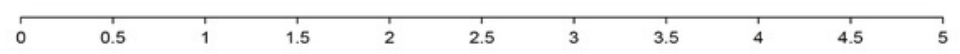

Total points

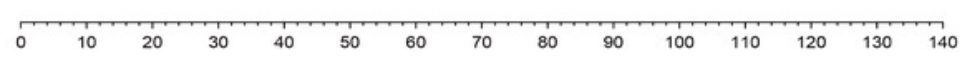

1-year survival

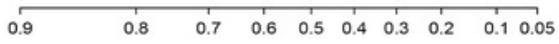

3-year survival

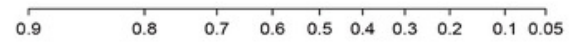

5-year survival

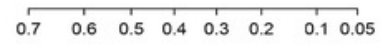

B
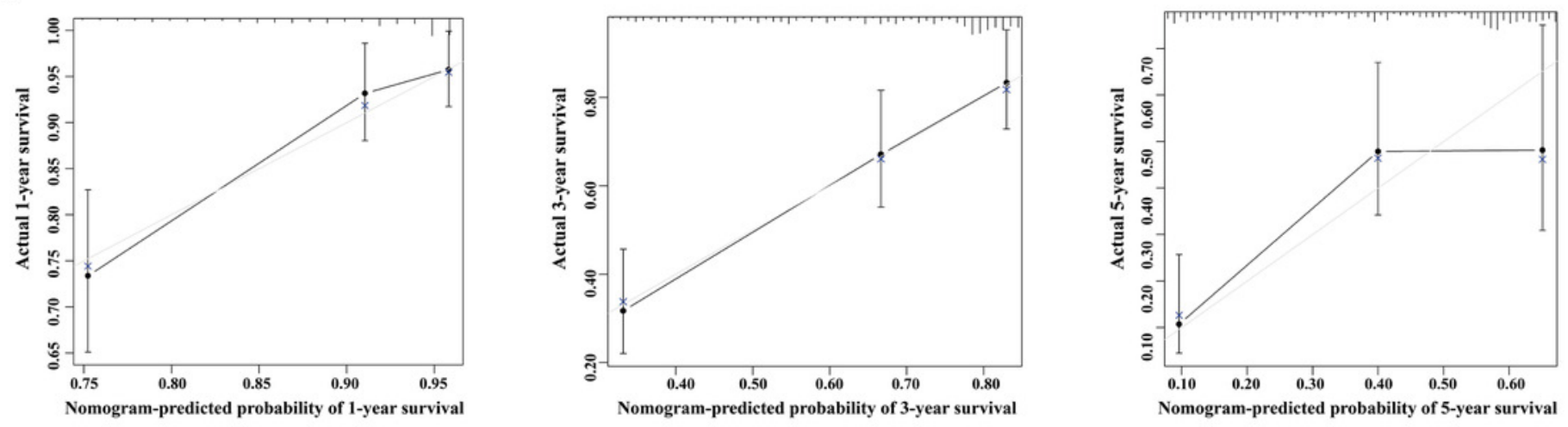

C

1-year AUC
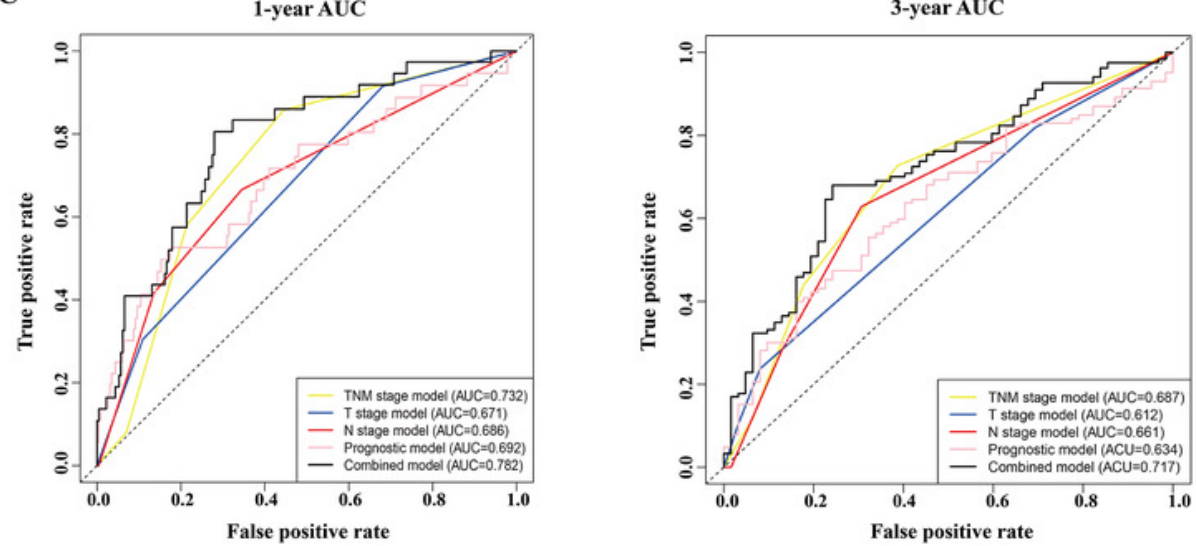

5-year AUC

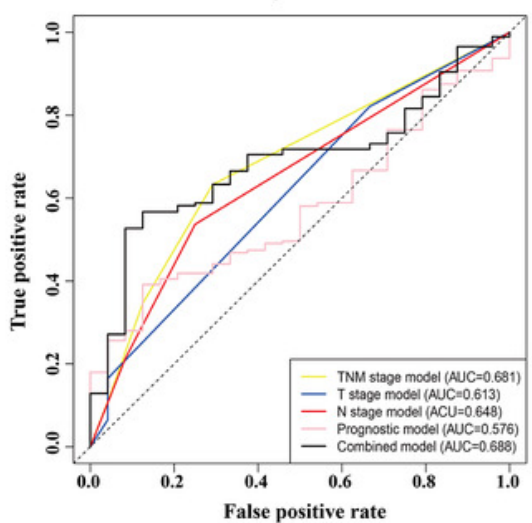




\section{Figure 7}

The representative enriched metabolism-related KEGG pathways in the TCGA dataset by GSEA.

(A) The top five significantly representative enriched metabolism-related KEGG pathways in the high-risk group. (B) The top five significantly representative enriched metabolism-related KEGG pathways in the low-risk group. Related parameters for the ten representative enriched metabolism-related KEGG pathways are given in Table 3. GSEA, Gene Set Enrichment Analysis; KEGG, Kyoto Encyclopedia of Genes and Genomes; TCGA, The Cancer Genome Atlas. 
$\mathbf{A}$
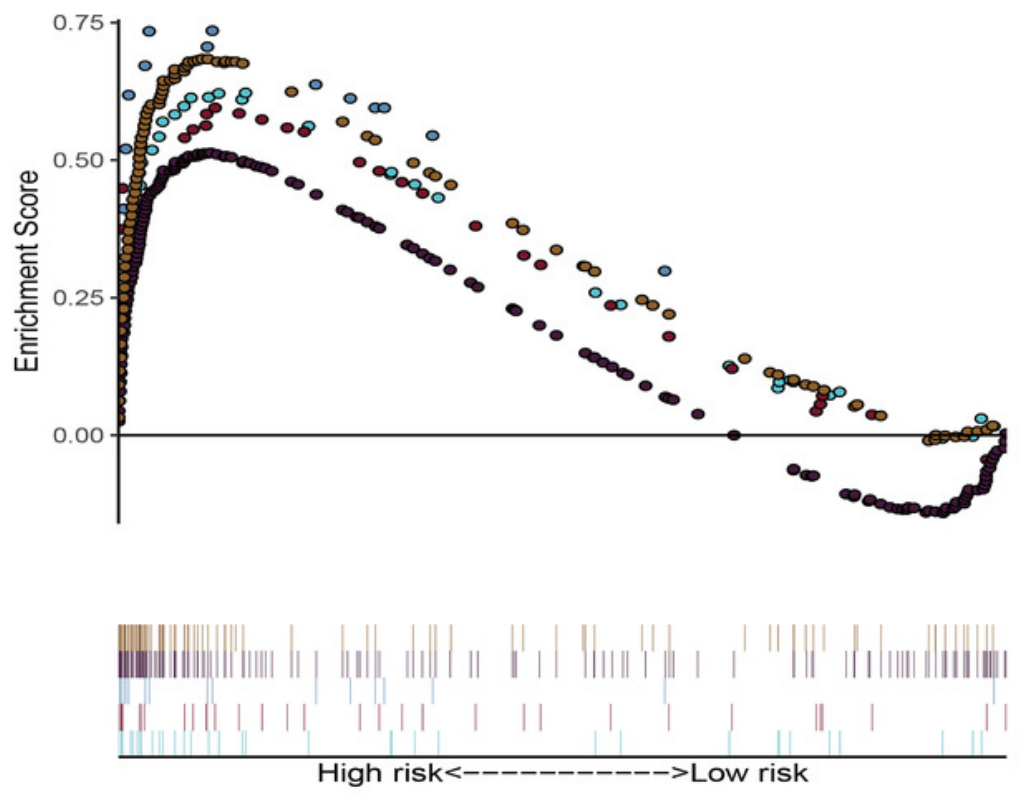

B

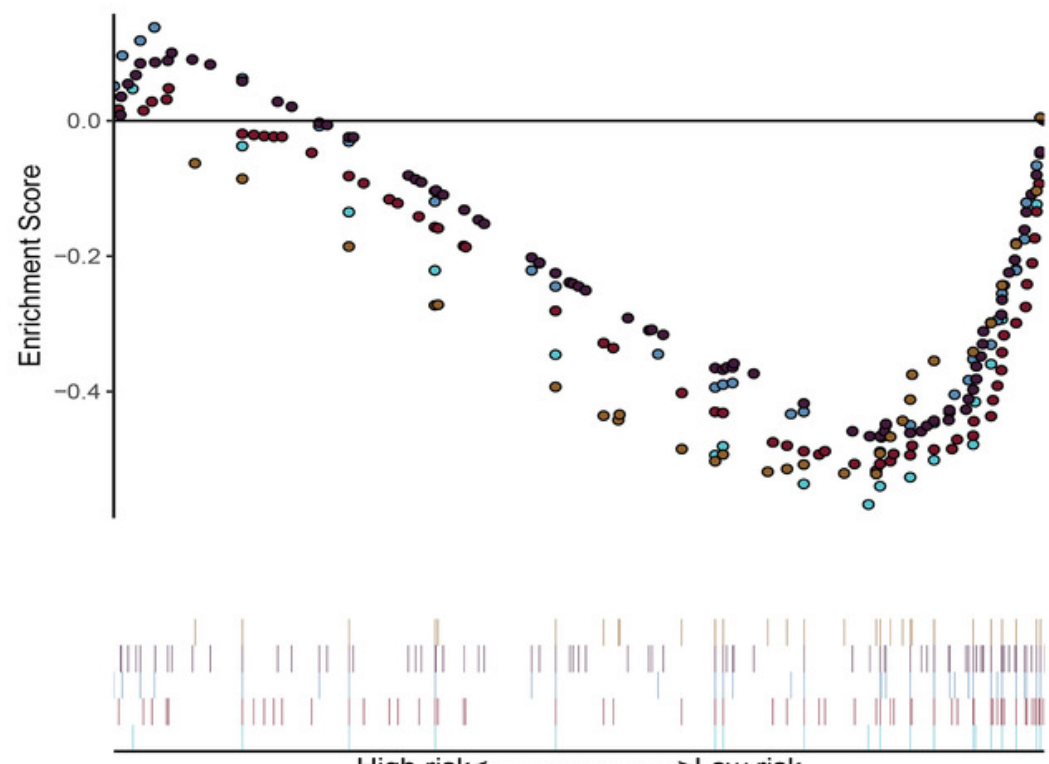

\section{High risk}

- KEGG_CYSTEINE_AND_METHIONINE_METABOLISM

- KEGG_FRUCTOSE_AND_MANNOSE_METABOLISM

- KEGG_GLYOXYLATE_AND_DICARBOXYLATE_METABOLISM

- KEGG_PURINE_METABOLISM

- KEGG_PYRIMIDINE_METABOLISM

\section{Low risk}

- KEGG_ALPHA_LINOLENIC_ACID_METABOLISM

- KEGG_ARACHIDONIC_ACID_METABOLISM

- KEGG_ETHER_LIPID_METABOLISM

- KEGG_GLYCEROPHOSPHOLIPID_METABOLISM

- KEGG_LINOLEIC_ACID_METABOLISM

High risk<-------------> Low risk 


\section{Figure 8}

mRNA expression levels of the six prognostic genes from online databases.

(A) mRNA expression levels of the six genes in the Oncomine database

(http://www.oncomine.org/). The threshold is shown at the bottom ( $P$ value $<0.001$ and fold change $>2$ were utilized for screening). The figure in the colored cell represents the number of datasets complying with the threshold. The red cells indicate that the genes were overexpressed in the cancer, while the blue cells indicate that the genes were overexpressed in the normal tissues. (B) Comparisons of the mRNA expression levels of the six genes between LUAD and normal tissues in the combined LUAD datasets from the Oncomine database. PFKP, phosphofructokinase platelet; PKM, pyruvate kinase muscle; TPI1, triosephosphate isomerase $1 ;$ LDHA, lactate dehydrogenase A; PTGES, prostaglandin E synthase; TYMS, thymidylate synthase; LUAD, lung adenocarcinoma. 
A

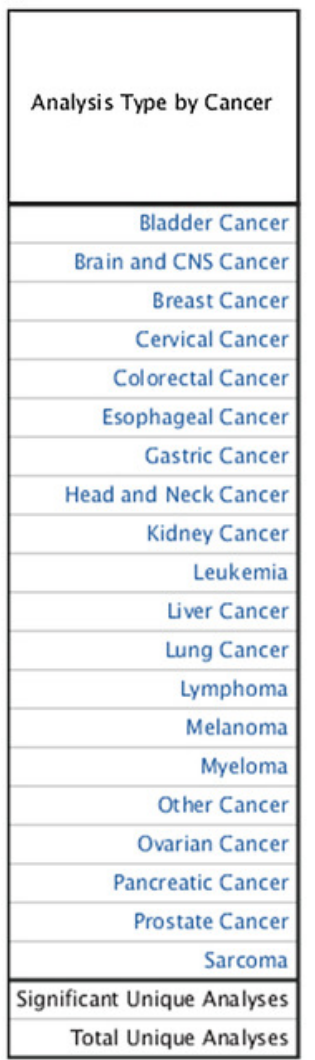

PFKP

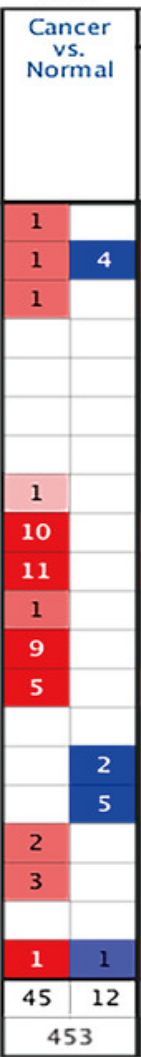

PKM

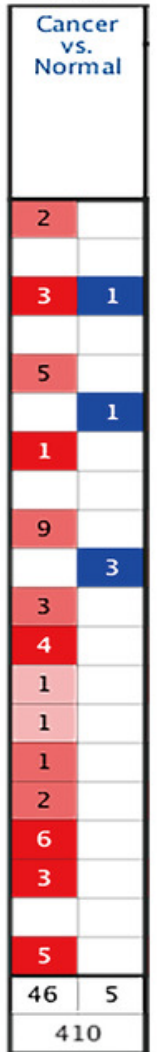

TPI1

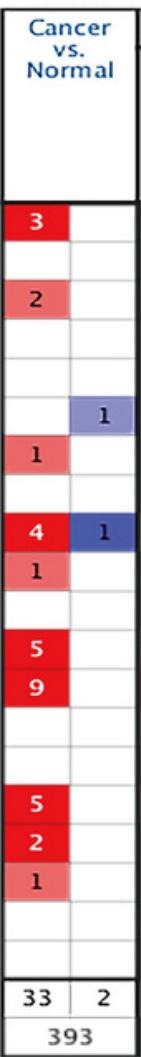

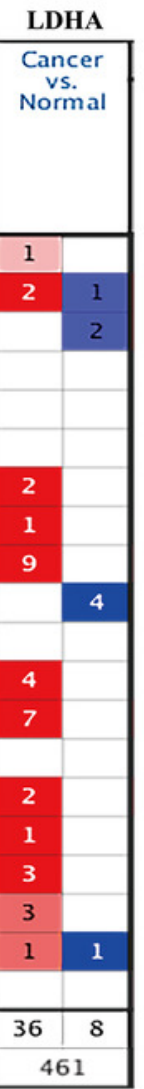

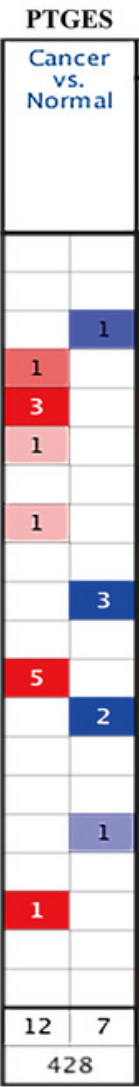

TYMS

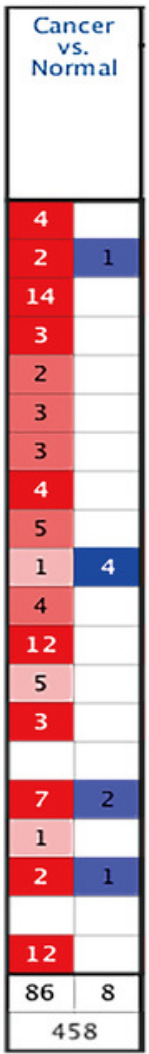

\section{$\square^{1} \square^{10} \square \square^{10} \square^{5} \square^{1}$}

THRESHOLD (P-VALUE: 0.001; FOLD CHANGE: 2; GENE RANK: Top 10\%)

Cell color is determined by the best gene rank percentile for the analysis within the cell. NOTE: An analysis may be counted in more than one cancer type.

B

Comparison of PFKP Across 5 Analyses

$$
\text { Over-expression }
$$

Median Rank p-Value Gene

$280.0 \quad 1.64 \mathrm{E}-17$ PFKP

\section{\begin{tabular}{|l|l|l|l|l|}
\hline 1 & 2 & 3 & 4 & 5 \\
\hline
\end{tabular}}

Legend

1. Lung Adenocarcinoma vs. Normal 4. Lung Adenocarcinoma vs. Normal

Garber Lung, Proc Natl Acad Sci U SA, Selamat Lung, Genome Res, 2012

2001 5. Lung Adenocarcinoma vs. Normal

2. Lung Adenocarcinoma vs. Normal

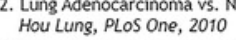

3. Lung Adenocarcinoma vs. Normal

Landi Lung, PLOS ONE, 2008

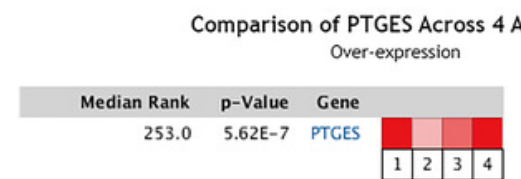

Legend

$\begin{array}{ll}\text { 1. Lung Adenocarcinoma vs. Normal } & \text { 3. Lung Adenocarcinoma vs. Normal }\end{array}$

Garber Lung, Proc Natl Acad Sci U S A, Selamat Lung, Genome Res, 2012

2001 2. Lung Adenocarcinoma vs. Normal $\quad$ Stearman Lung, Am J Pathol, 2005
Hou Lung, PLoS One, 2010

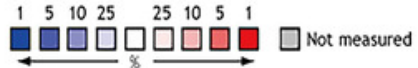

The rank for a gene is the median rank for that gene across each of the analyses.

The p-Value for a gene is its p-Value for the median-ranked analysis.
Comparison of LDHA Across 2 Analyses Over-expression

Median Rank p-Value Gene

$85.5 \quad 3.15 \mathrm{E}-4$ LDHA

\section{\begin{tabular}{|l|l|}
\hline 1 & 2 \\
\hline
\end{tabular}}

Legend

1. Lung Adenocarcinoma vs. Normal

Selamat Lung, Genome Res, 2012

2. Lung Adenocarcinoma vs. Normal

Yamagata Lung, Clin Cancer Res, 2003

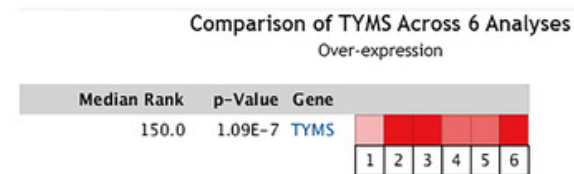

Legend

1. Lung Adenocarcinoma vs. Normal 4. Lung Adenocarcinoma vs. Normal

Beer Lung, Nat Med, 2002 Selamat Lung, Genome Res, 2012

2. Lung Adenocarcinoma vs. Normal $\quad$ 5. Lung Adenocarcinoma vs. Normal

Hou Lung, PLoS One, 2010 Stearman Lung, Am J Pathol, 2005

3. Lung Adenocarcinoma vs. Normal $\quad 6$. Lung Adenocarcinoma vs. Normal

$\begin{array}{ll}\text { Landi Lung, PLOS ONE, } 2008 & \text { Su Lung, BMC Genomics, } 2007\end{array}$ 


\section{Figure 9}

mRNA expression levels of the six prognostic genes extracted from online database.

The mRNA expression levels of the six genes in different tumour types from the TIMER database ( http://cistrome.shinyapps.io/timer/) $\left(* P<0.05,{ }^{*} * P<0.01,{ }^{* * * P}<0.001\right)$. PFKP, phosphofructokinase platelet; $P K M$, pyruvate kinase muscle; TPI1, triosephosphate isomerase 1; LDHA, lactate dehydrogenase A; PTGES, prostaglandin E synthase; TYMS, thymidylate synthase. 

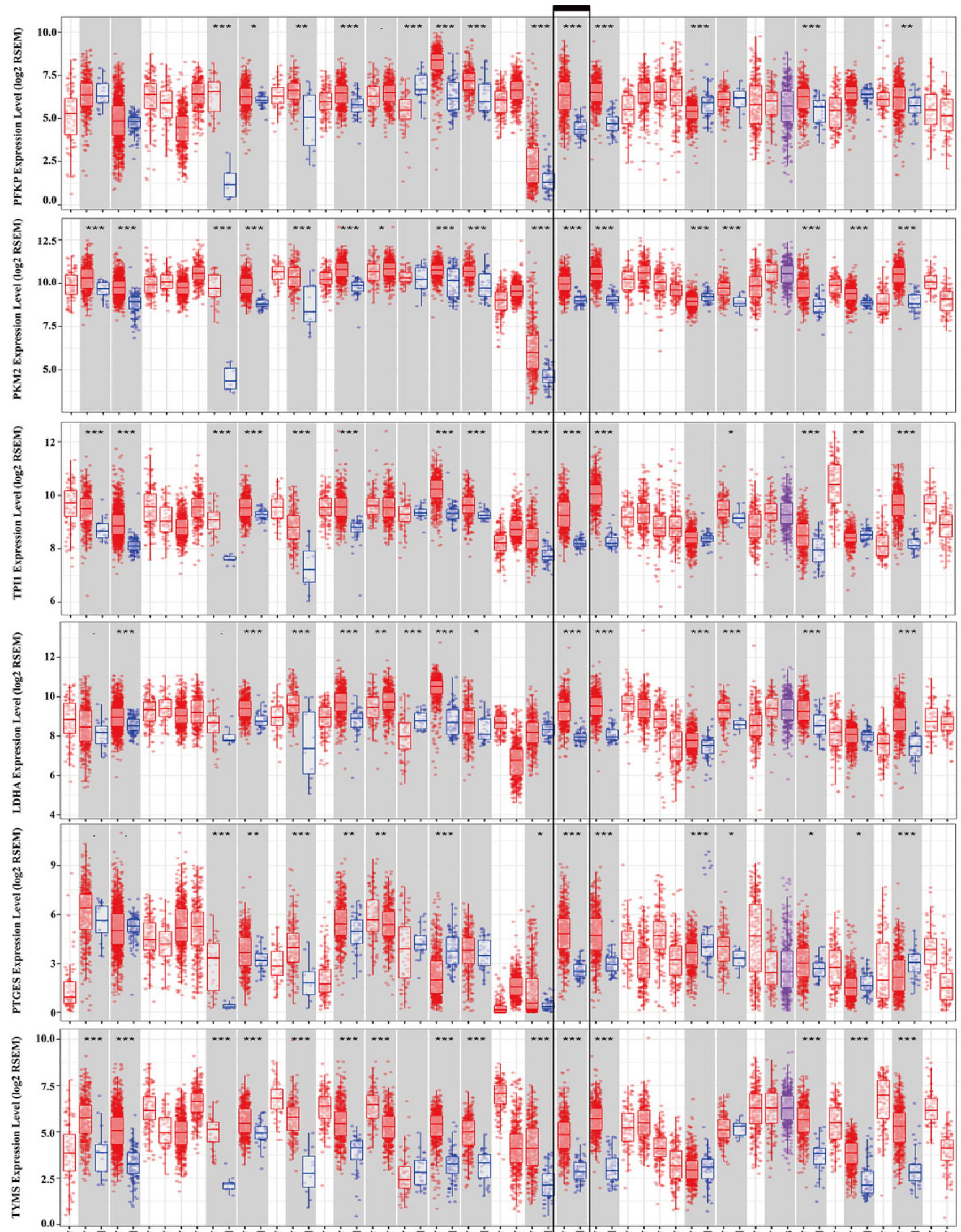

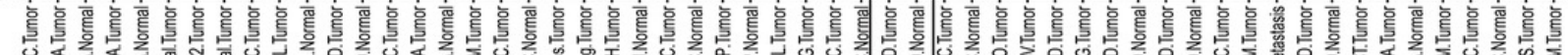

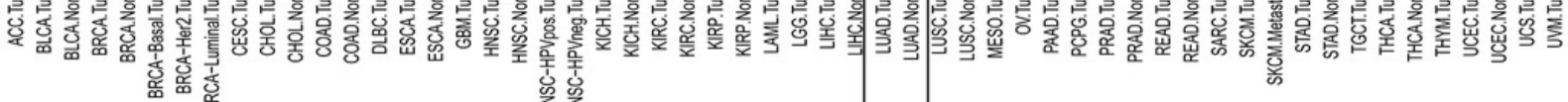




\section{Figure 10}

Protein expression levels and genetic alterations of the corresponding six prognostic genes obtained from online databases.

(A) The representative immunohistochemistry images of the protein expression of the six genes in the normal lung tissues and LUAD tissues from the Human Protein Atlas database. (http://www.proteinatlas.org/) (B) The percentage of protein expression levels in the normal lung tissues and LUAD tissues analysed based on the Human Protein Atlas database. AntiPFKP antibody is HPA018257; anti-PKM antibody is CAB019421; anti-TPI1 antibody is HPA053568; anti-LDHA antibody is CAB069404; anti-PTGES is HPA045064; anti-TYMS antibody is CAB002784. (C) Genetic alterations of the six genes in 230 LUAD patients / samples (TCGA, Firehose Legacy). Data were obtained from the cBioportal for Cancer Genomics ( http://www.cbioportal.org/ ). PFKP, phosphofructokinase platelet; PKM, pyruvate kinase muscle; TPI1, triosephosphate isomerase 1; LDHA, lactate dehydrogenase A; PTGES, prostaglandin E synthase; TYMS, thymidylate synthase; TCGA, The Cancer Genome Atlas; LUAD, lung adenocarcinoma. 
A

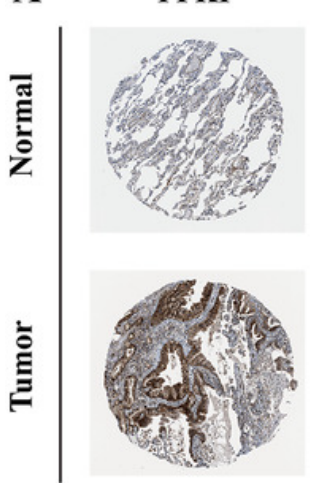

LDHA

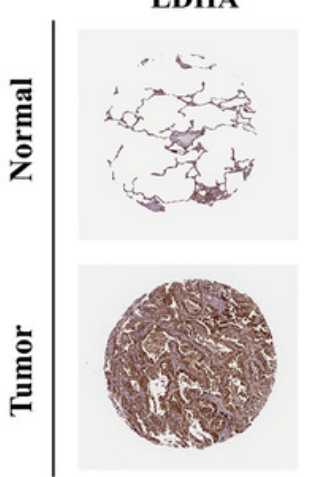

B

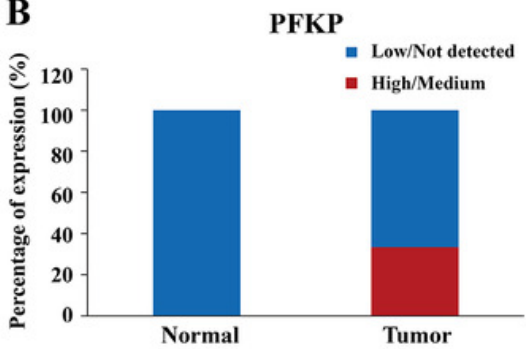

LDHA

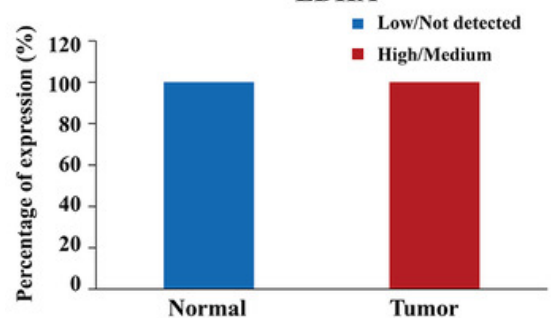

PKM

Patient id: $\mathbf{2 1 0 1}$ Staining: Not detected Intensity: Weak Quantity: $<25 \%$

Patient id: 3391 Staining: High Intensity: Strong

Patient id: 2208 Staining: Low Intensity: Moderate Quantity: $<25 \%$

Patient id: $\mathbf{4 8 8}$ Staining: High Intensity: Strong Quantity: $>75 \%$

Tumor

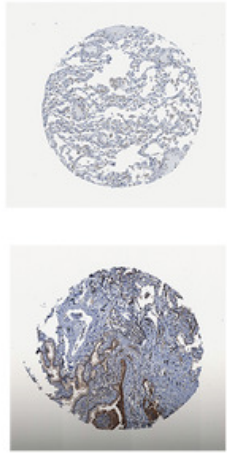

Patient id: $\mathbf{2 2 6 8}$ Staining: Low Intensity: Moderate Quantity: $<25 \%$

Patient id: 3391 Staining: High Intensity: Strong Quantity: $>75 \%$

PTGES

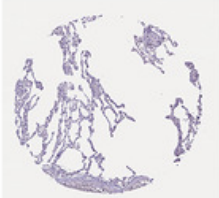

Staining: Not detected Intensity: Negative Quantity: None

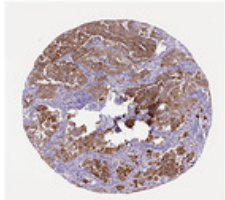

Patient id: 2003 Staining: Medium Intensity: Moderate Quantity: $>75 \%$

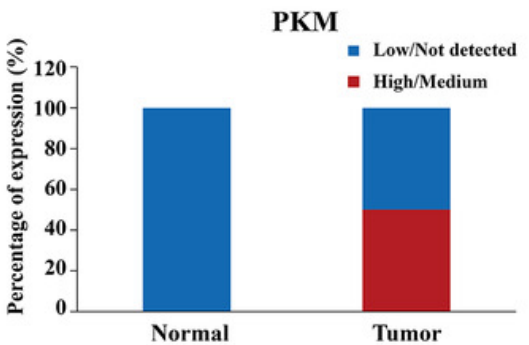

PTGES

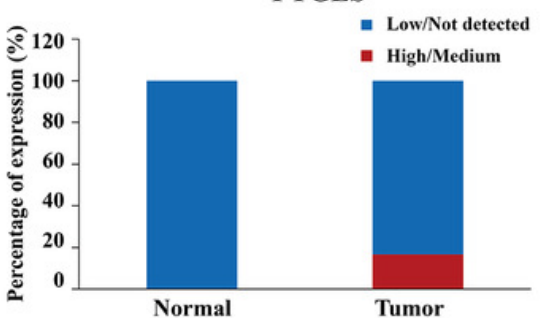

TPI1

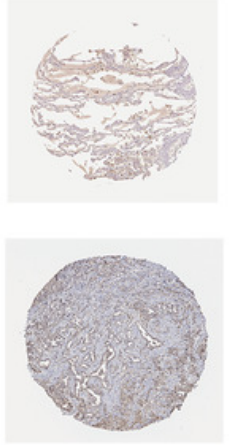

Patient id: 2208

Staining: Low

Intensity: Moderate

Quantity: $<\mathbf{2 5 \%}$

Patient id: $\mathbf{4 2 0 8}$

Staining: Not detected Intensity: Weak Quantity: $<25 \%$

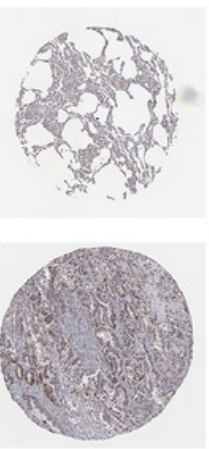

Patient id: 2268

Staining: Not detected Intensity: Negative

Quantity: None

Patient id: 1847

Staining: Medium Intensity: Moderate

Quantity: $\mathbf{7 5} \%-\mathbf{2 5} \%$

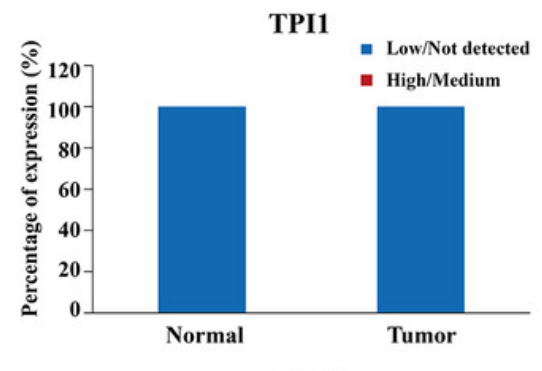

TYMS

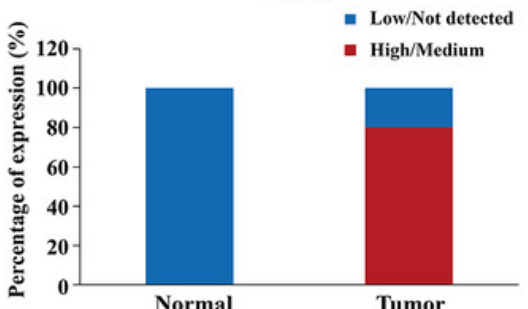

C Tumor samples samples with sequencing and copy number variation data ( 230 patients/samples)

PFKP 2.6\%

PKM 1.3\%

TPI1 5\%

LDHA $2.6 \%$

PTGES 0.9\%

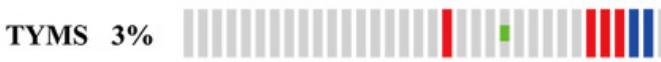

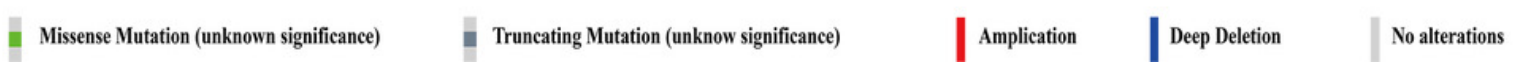


Table $\mathbf{1}$ (on next page)

Clinical characteristics of the included datasets 
1

\begin{tabular}{|c|c|c|}
\hline Characteristics & $\begin{array}{l}\text { TCGA (n, \%) } \\
(\mathrm{n}=454)\end{array}$ & $\begin{array}{l}\text { GSE68465 (n, \%) } \\
(\mathrm{n}=439)\end{array}$ \\
\hline \multicolumn{3}{|l|}{ Age } \\
\hline$<60$ & $133(29.3 \%)$ & $128(29.2 \%)$ \\
\hline$\geq 60$ & $321(70.7 \%)$ & $311(70.8 \%)$ \\
\hline NA & $0(0.0 \%)$ & $0(0.0 \%)$ \\
\hline \multicolumn{3}{|l|}{ Gender } \\
\hline Female & $248(54.6 \%)$ & $218(49.7 \%)$ \\
\hline Male & $206(45.4 \%)$ & $221(50.3 \%)$ \\
\hline NA & $0(0.0 \%)$ & $0(0.0 \%)$ \\
\hline \multicolumn{3}{|l|}{ Grade } \\
\hline G1 & $0(0.0 \%)$ & $60(13.7 \%)$ \\
\hline $\mathrm{G} 2$ & $0(0.0 \%)$ & $206(46.9 \%)$ \\
\hline G3 & $0(0.0 \%)$ & $166(37.8 \%)$ \\
\hline NA & $454(100 \%)$ & $7(1.6 \%)$ \\
\hline \multicolumn{3}{|l|}{ TNM stage } \\
\hline I & $243(53.5 \%)$ & \\
\hline II & $105(23.1 \%)$ & \\
\hline III & $74(16.3 \%)$ & \\
\hline IV & $24(5.3 \%)$ & \\
\hline NA & $8(1.8 \%)$ & $439(100 \%)$ \\
\hline \multicolumn{3}{|l|}{ T stage } \\
\hline $\mathrm{T} 1$ & $156(34.4 \%)$ & $150(34.2 \%)$ \\
\hline $\mathrm{T} 2$ & $240(52.9 \%)$ & $248(56.5 \%)$ \\
\hline T3 & $37(8.1 \%)$ & $28(6.4 \%)$ \\
\hline $\mathrm{T} 4$ & $18(4.0 \%)$ & $11(2.5 \%)$ \\
\hline $\mathrm{Tx}$ & $3(0.7 \%)$ & $2(0.5 \%)$ \\
\hline \multicolumn{3}{|l|}{$\mathrm{N}$ stage } \\
\hline No & $291(64.1 \%)$ & $297(67.7 \%)$ \\
\hline N1 & $86(18.9 \%)$ & $87(19.8 \%)$ \\
\hline $\mathrm{N} 2$ & $64(14.1 \%)$ & $52(11.8 \%)$ \\
\hline N3 & $2(0.4 \%)$ & $0(0.0 \%)$ \\
\hline $\mathrm{Nx}$ & $11(2.4 \%)$ & $3(0.7 \%)$ \\
\hline \multicolumn{3}{|l|}{ M stage } \\
\hline M0 & $305(67.2 \%)$ & $439(100 \%)$ \\
\hline M1 & $23(5.1 \%)$ & $0(0.0 \%)$ \\
\hline $\mathrm{Mx}$ & $126(27.8 \%)$ & $0(0.0 \%)$ \\
\hline \multicolumn{3}{|l|}{ Survival status } \\
\hline Alive & $300(66.1 \%)$ & $206(46.9 \%)$ \\
\hline Dead & $154(33.9 \%)$ & $233(53.1 \%)$ \\
\hline
\end{tabular}


2 Note:

3 TCGA, The Cancer Genome Atlas.

4 
Table 2 (on next page)

Prognostic values for the six-gene metabolic signature in 454 LUAD patients. 


\begin{tabular}{llllll}
\hline Gene & Coef & HR & HR.95L & HR.95H & P value \\
\hline PFKP & 0.000050 & 1.009949 & 1.004184 & 1.015747 & 0.0007 \\
PKM & 0.001734 & 1.005149 & 1.002545 & 1.007759 & 0.000104 \\
TPI1 & 0.000384 & 1.003352 & 1.001479 & 1.005229 & 0.000448 \\
LDHA & 0.003792 & 1.005663 & 1.003774 & 1.007556 & 0.00000000396 \\
PTGES & 0.002922 & 1.008392 & 1.003408 & 1.0134 & 0.000946 \\
TYMS & 0.024904 & 1.033141 & 1.01572 & 1.050861 & 0.000172 \\
\hline
\end{tabular}

1 Note:

2 LUAD, lung adenocarcinoma; HR, hazard ratio; CI, confidence interval. 


\section{Table 3(on next page)}

The results of the ten representative enriched metabolism-related KEGG pathways analysed by GSEA. 


\begin{tabular}{|c|c|c|c|c|c|}
\hline Pathway & Size & $\mathbf{E S}$ & NES & $\begin{array}{l}\text { NOM } \\
p \text {-val }\end{array}$ & $\begin{array}{l}\text { FDR } \\
q \text {-val }\end{array}$ \\
\hline \multicolumn{6}{|l|}{ High risk } \\
\hline KEGG_CYSTEINE_AND_METHIONINE_METABOLISM & 34 & 0.62 & 1.98 & 0.00 & 0.006 \\
\hline KEGG_FRUCTOSE_AND_MANNOSE_METABOLISM & 33 & 0.60 & 1.95 & 0.002 & 0.007 \\
\hline $\begin{array}{l}\text { KEGG_GLYOXYLATE_AND_DICARBOXYLATE_ } \\
\text { METABOLISM }\end{array}$ & 16 & 0.74 & 1.89 & 0.002 & 0.013 \\
\hline KEGG_PURINE_METABOLISM & 157 & 0.51 & 2.02 & 0.000 & 0.005 \\
\hline $\begin{array}{l}\text { KEGG_PYRIMIDINE_METABOLOSM } \\
\text { Low risk }\end{array}$ & 98 & 0.68 & 2.36 & 0.000 & 0.000 \\
\hline KEGG_ALPHA_LINOLENIC_ACID_METABOLISM & 19 & -0.60 & -1.82 & 0.002 & 0.113 \\
\hline KEGG_ARACHIDONIC_ACID_METABOLISM & 58 & -0.53 & -1.86 & 0.000 & 0.101 \\
\hline KEGG_ETHER_LIPID_METABOLISM & 33 & -0.51 & -1.73 & 0.011 & 0.129 \\
\hline KEGG_GLYCEROPHOSPHOLIPID_METABOLISM & 77 & -0.48 & -1.91 & 0.002 & 0.127 \\
\hline KEGG_LINOLEIC_ACID_METABOLISM & 29 & -0.55 & -1.77 & 0.008 & 0.138 \\
\hline
\end{tabular}

Note:

KEGG, Kyoto Encyclopedia of Genes and Genomes; GSEA, Gene Set Enrichment Analysis; ES, enrichment score; NOM $p$-val, nominal $p$-value; FDR $q$-val, false discovery rate $q$-value; NES, normalized enrichment score. 


\section{Table 4 (on next page)}

Comparison of mRNA expression levels of the six genes between LUAD and normal tissues from the Oncomine database. 


\begin{tabular}{|c|c|c|c|c|c|}
\hline Gene & $\begin{array}{l}\text { Analysis Type of lung cancer vs. } \\
\text { normal }\end{array}$ & t-Test & $\begin{array}{l}\text { Fold } \\
\text { change }\end{array}$ & $P$ value & References \\
\hline \multirow[t]{7}{*}{ PFKP } & LUAD (n=40) vs. Normal $(n=6)$ & 7.146 & 3.469 & $7.56 \mathrm{E}-8$ & Garber et al., 2001 \\
\hline & LUAD $(n=58)$ vs. Normal $(n=49)$ & 11.177 & 2.685 & $4.35 \mathrm{E}-19$ & Landi et al., 2008 \\
\hline & LUAD $(n=45)$ vs. Normal $(n=65)$ & 7.946 & 2.536 & 3.39E-11 & Hou et al., 2010 \\
\hline & LUAD $(n=58)$ vs. Normal $(n=58)$ & 10.910 & 2.883 & $1.64 \mathrm{E}-17$ & Selamat et al., 2012 \\
\hline & LUAD $(n=20)$ vs. Normal $(n=19)$ & 5.277 & 3.149 & $9.37 \mathrm{E}-6$ & Stearman et al., 2005 \\
\hline & Comparison of PFKP expression across 5 & - & - & $1.64 \mathrm{E}-17$ & - \\
\hline & Analysis between LUAD and Normal & & & & \\
\hline PKM & LUAD $(\mathrm{n}=58)$ vs. Normal $(\mathrm{n}=58)$ & 12.037 & 2.551 & $3.56 \mathrm{E}-20$ & Selamat et al., 2012 \\
\hline TPI1 & LUAD ( $n=40)$ vs. Normal $(n=6)$ & 4.929 & 2.283 & $4.03 \mathrm{E}-4$ & Garber et al., 2001 \\
\hline \multirow[t]{4}{*}{ LDHA } & LUAD ( $n=9)$ vs. Normal $(n=3)$ & 4.502 & 4.037 & $6.29 \mathrm{E}-4$ & Yamagata et al. 2003 \\
\hline & LUAD $(n=58)$ vs. Normal $(n=58)$ & 11.533 & 2.179 & $1.59 \mathrm{E}-19$ & Selamat et al., 2012 \\
\hline & Comparison of LDHA expression across & - & - & $3.15 \mathrm{E}-4$ & - \\
\hline & 2 Analysis between LUAD and Normal & & & & \\
\hline \multirow[t]{6}{*}{ PTGES } & LUAD $(n=20)$ vs. Normal $(n=19)$ & 9.332 & 5.883 & $1.54 \mathrm{E}-11$ & Stearman et al., 2005 \\
\hline & LUAD $(n=40)$ vs. Normal $(n=6)$ & 6.690 & 4.969 & $1.12 \mathrm{E}-6$ & Garber et al., 2001 \\
\hline & LUAD $(\mathrm{n}=58)$ vs. Normal $(\mathrm{n}=58)$ & 10.267 & 2.179 & $5.58 \mathrm{E}-16$ & Selamat et al., 2012 \\
\hline & LUAD $(n=45)$ vs. Normal $(n=65)$ & 6.513 & 2.170 & $6.22 \mathrm{E}-9$ & Hou et al., 2010 \\
\hline & Comparison of PTGES expression across & - & - & $5.62 \mathrm{E}-7$ & - \\
\hline & 4 Analysis between LUAD and Normal & & & & \\
\hline \multirow[t]{8}{*}{ TYMS } & LUAD $(n=45)$ vs. Normal $(n=65)$ & 9.322 & 3.929 & $6.92 \mathrm{E}-15$ & Hou et al., 2010 \\
\hline & LUAD $(n=27)$ vs. Normal $(n=30)$ & 7.395 & 3.016 & $2.40 \mathrm{E}-9$ & Su et al., 2007 \\
\hline & LUAD $(n=58)$ vs. Normal $(n=49)$ & 11.169 & 2.797 & $9.86 \mathrm{E}-20$ & Landi et al., 2008 \\
\hline & LUAD $(n=20)$ vs. Normal $(n=19)$ & 6.509 & 2.118 & $2.18 \mathrm{E}-7$ & Stearman et al., 2005 \\
\hline & LUAD $(n=86)$ vs. Normal $(n=10)$ & 4.191 & 2.158 & $3.05 \mathrm{E}-4$ & Beer et al., 2002 \\
\hline & LUAD $(\mathrm{n}=58)$ vs. Normal $(\mathrm{n}=58)$ & 8.565 & 2.040 & $3.35 \mathrm{E}-13$ & Selamat et al., 2012 \\
\hline & Comparison of TYMS expression across & - & - & $1.09 \mathrm{E}-7$ & - \\
\hline & 6 Analysis between LUAD and Normal & & & & \\
\hline
\end{tabular}

1 Note:

2 Owing to only one dataset meeting the screening criteria, the comparison of PKM or TPI1 expression in LUAD and normal has not been built based on

3 the combined LUAD datasets. $P$ value $<0.001$ and fold change $>2$ were utilized for screening. LUAD, lung adenocarcinoma. 


\section{Table 5 (on next page)}

Protein expression levels of the six prognostic genes in the normal lung tissues and LUAD tissues obtained from the Human Protein Atlas database. 


\begin{tabular}{llll}
\hline Gene name & Tissue type & $\begin{array}{l}\text { Patients in high/medium } \\
\text { staining n }(\%)\end{array}$ & $\begin{array}{l}\text { Patients in low/not detected } \\
\text { staining n (\%) }\end{array}$ \\
\hline PFKP & Normal & $0(0 \%)$ & $3(100 \%)$ \\
& Tumor & $2(33.33 \%)$ & $4(66.67 \%)$ \\
PKM & Normal & $0(0 \%)$ & $3(100 \%)$ \\
& Tumor & $3(50 \%)$ & $3(50 \%)$ \\
TPI1 & Normal & $0(0 \%)$ & $3(100 \%)$ \\
& Tumor & $0(0 \%)$ & $3(100 \%)$ \\
\multirow{2}{*}{ LDHA } & Normal & $0(0 \%)$ & $3(100 \%)$ \\
& Tumor & $7(100 \%)$ & $0(0 \%)$ \\
PTGES & Normal & $0(0 \%)$ & $3(100 \%)$ \\
& Tumor & $1(16.67 \%)$ & $5(83.33 \%)$ \\
\multirow{2}{*}{ TYMS } & Normal & $0(0 \%)$ & $3(100 \%)$ \\
& Tumor & $4(80 \%)$ & $1(20 \%)$ \\
\hline
\end{tabular}

1 Note:

2 LUAD, lung adenocarcinoma. 